\title{
Search for production of Higgs boson pairs in the four b quark final state using large-area jets in proton-proton collisions at $\sqrt{s}=13 \mathrm{TeV}$
}

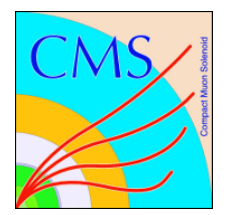

\section{The CMS collaboration}

\author{
E-mail: cms-publication-committee-chair@cern.ch
}

\begin{abstract}
A search is presented for pair production of the standard model Higgs boson using data from proton-proton collisions at a centre-of-mass energy of $13 \mathrm{TeV}$, collected by the CMS experiment at the CERN LHC in 2016, and corresponding to an integrated luminosity of $35.9 \mathrm{fb}^{-1}$. The final state consists of two b quark-antiquark pairs. The search is conducted in the region of phase space where one pair is highly Lorentz-boosted and is reconstructed as a single large-area jet, and the other pair is resolved and is reconstructed using two b-tagged jets. The results are obtained by combining this analysis with another from CMS looking for events with two large jets. Limits are set on the product of the cross sections and branching fractions for narrow bulk gravitons and radions in warped extradimensional models having a mass in the range $750-3000 \mathrm{GeV}$. The resulting observed and expected upper limits on the non-resonant Higgs boson pair production cross section correspond to 179 and 114 times the standard model value, respectively, at $95 \%$ confidence level. The existence of anomalous Higgs boson couplings is also investigated and limits are set on the non-resonant Higgs boson pair production cross sections for representative coupling values.
\end{abstract}

Keywords: Beyond Standard Model, Hadron-Hadron scattering (experiments), Higgs physics, Jets

ARXIV EPRINT: 1808.01473 


\section{Contents}

1 Introduction 1

2 The CMS detector and event reconstruction 3

3 Event simulation $\quad 4$

$\begin{array}{lll}4 & \text { Event selection } & 6\end{array}$

$\begin{array}{llr}5 & \text { Background estimation } & 11\end{array}$

$\begin{array}{llr}6 & \text { Systematic uncertainties } & 13\end{array}$

$\begin{array}{lll}7 & \text { Results } & 14\end{array}$

$\begin{array}{lll}8 & \text { Summary } & 18\end{array}$

$\begin{array}{lr}\text { The CMS collaboration } & 26\end{array}$

\section{Introduction}

In the standard model (SM), Higgs boson (H) [1-3] pair-production can occur through several subprocesses and is sensitive to the Higgs boson self-coupling. In proton-proton (pp) collisions at the CERN LHC, the SM HH production cross section is mainly due to the gluon-gluon fusion subprocess, which proceeds via an internal fermion loop dominated by the top quark, t. At a centre-of-mass energy of $13 \mathrm{TeV}$, this cross section is $33.5_{-2.8}^{+2.5} \mathrm{fb}$ [ 4 6], which is too small to be observable using the current data. However, many beyond the standard model (BSM) theories predict higher rates of Higgs boson pair production. The rate could be increased through the production of a massive BSM resonance $\mathrm{X}$, which subsequently decays to a Higgs boson pair $(\mathrm{X} \rightarrow \mathrm{HH})$ [7], a process that could be observable at the LHC. If the resonance mass $m_{\mathrm{X}}$ is too large for $\mathrm{X}$ to be directly produced in $\mathrm{pp}$ interactions, the particle could manifest itself through off-shell effects, leading to anomalous couplings of the $\mathrm{H}$ boson to the SM particles, including the HH self-interaction [8]. Thus, BSM effects may modify the HH differential and integral production cross sections, making this process observable with current data.

Models with a warped extra dimension (WED), as proposed by Randall and Sundrum [7], are among those BSM scenarios that predict the existence of resonances with large couplings to the SM Higgs boson, such as the spin-0 radion [9-11] and the spin-2 first Kaluza-Klein (KK) excitation of the graviton [12-14]. The WED models postulate an additional spatial dimension $l$ compactified between two four-dimensional hypersurfaces known 
as the branes, with the region between, the bulk, warped by an exponential metric $\kappa l$, where $\kappa$ is the warp factor [15]. A value of $\kappa l \sim 35$ fixes the mass hierarchy between the Planck scale $M_{\mathrm{Pl}}$ and the electroweak scale [7]. One of the parameters of the model is $\kappa / \overline{M_{\mathrm{Pl}}}$, where $\overline{M_{\mathrm{Pl}}} \equiv M_{\mathrm{Pl}} / \sqrt{8 \pi}$. The ultraviolet cutoff scale of the model $\Lambda_{\mathrm{R}} \equiv \sqrt{6} \mathrm{e}^{-\kappa l} \overline{M_{\mathrm{Pl}}}[9]$ is another parameter, and is expected to be near the $\mathrm{TeV}$ scale.

In the absence of new resonances coupling to the Higgs boson, the gluon fusion Higgs boson pair production subprocess can still be enhanced by BSM contributions to the coupling parameters of the Higgs boson and the SM fields [16]. The SM production rate of $\mathrm{HH}$ through gluon fusion is determined by the Yukawa coupling of the Higgs boson to the top quark $y_{\mathrm{t}}^{\mathrm{SM}}$ and the Higgs boson self-coupling $\lambda_{\mathrm{HH}}^{\mathrm{SM}}=m_{\mathrm{H}}^{2} / 2 v^{2}$. Here, $m_{\mathrm{H}}=125 \mathrm{GeV}$ is the Higgs boson mass $[17,18]$ and $v=246 \mathrm{GeV}$ is the vacuum expectation value of the Higgs field. Deviations from the SM values of these two coupling parameters can be expressed as $\kappa_{\lambda} \equiv \lambda_{\mathrm{HH}} / \lambda_{\mathrm{HH}}^{\mathrm{SM}}$ and $\kappa_{\mathrm{t}} \equiv y_{\mathrm{t}} / y_{\mathrm{t}}^{\mathrm{SM}}$, respectively. Depending on the BSM scenario, other couplings not present in the SM may also exist and can be described by dimension- 6 operators in the framework of an effective field theory by the Lagrangian [19]:

$$
\begin{aligned}
\mathcal{L}_{\mathrm{H}}= & \frac{1}{2} \partial_{\mu} \mathrm{H} \partial^{\mu} \mathrm{H}-\frac{1}{2} m_{\mathrm{H}}^{2} \mathrm{H}^{2}-\kappa_{\lambda} \lambda_{\mathrm{HH}}^{\mathrm{SM}} v \mathrm{H}^{3}-\frac{m_{\mathrm{t}}}{v}\left(v+\kappa_{\mathrm{t}} \mathrm{H}+\frac{c_{2}}{v} \mathrm{HH}\right)\left(\overline{\mathrm{t}}_{\mathrm{L}} \mathrm{t}_{\mathrm{R}}+\text { h.c. }\right) \\
& +\frac{1}{4} \frac{\alpha_{s}}{3 \pi v}\left(c_{\mathrm{g}} \mathrm{H}-\frac{c_{2 \mathrm{~g}}}{2 v} \mathrm{HH}\right) G^{\mu \nu} G_{\mu \nu} .
\end{aligned}
$$

The anomalous couplings and the corresponding parameters in this Lagrangian are: the contact interaction between a pair of Higgs bosons and a pair of top quarks $\left(c_{2}\right)$, the interaction between the Higgs boson and the gluon $\left(c_{\mathrm{g}}\right)$, and the interaction between a pair of Higgs bosons and a pair of gluons $\left(c_{2 \mathrm{~g}}\right)$. The couplings with CP-violation and the interactions of the Higgs boson with light SM and BSM particles are not considered. The Lagrangian models the effects of BSM scenarios with a scale that is beyond the direct LHC reach. This five-parameter space of BSM Higgs couplings has constraints from measurements of single Higgs boson production and other theoretical considerations [20, 21].

Searches for HH production have been performed by the ATLAS [22-29] and CMS [3038] Collaborations using the LHC pp collision data at $\sqrt{s}=8$ and $13 \mathrm{TeV}$. A search targeting the high $m_{\mathrm{X}}$ range for a $\mathrm{KK}$ bulk graviton or a radion decaying to $\mathrm{HH}$, in the $\mathrm{b} \overline{\mathrm{b}} \mathrm{b} \overline{\mathrm{b}}$ final state, was published by the CMS Collaboration [39], in which two large-area jets are used to reconstruct the highly Lorentz-boosted Higgs bosons ("fully-merged" event topology). A similar search, focusing on a lower range of $m_{\mathrm{X}}$, was also performed by CMS [40], using events with four separate b quark jets. The configuration of a Higgs boson candidate as one large-area jet or as two separate smaller jets is dependent on the momentum of the Higgs boson [41].

In this paper, we improve upon the CMS search for high mass resonance (750 $\leq$ $m_{\mathrm{X}} \leq 3000 \mathrm{GeV}$ ) decaying to $\mathrm{HH} \rightarrow \mathrm{b} \overline{\mathrm{b}} \mathrm{b} \overline{\mathrm{b}}$ [39] by using "semi-resolved" events, i.e. those containing exactly one highly Lorentz-boosted Higgs boson while the other Higgs boson is required to have a lower boost. The data set corresponds to an integrated luminosity of $35.9 \mathrm{fb}^{-1}$ from pp collisions at $13 \mathrm{TeV}$. The more boosted Higgs boson is reconstructed using a large-area jet and the other is reconstructed from two separate b quark jets. The inclusion of the semi-resolved events leads to a significant improvement in the search sensitivity for 
resonances with $750 \leq m_{\mathrm{X}} \leq 2000 \mathrm{GeV}$. With the addition of the semi-resolved events, a signal from the non-resonant production of $\mathrm{HH}$ is also accessible using boosted topologies, since such production typically results in an $\mathrm{HH}$ invariant mass that is lower than that of a postulated resonance signal. For full sensitivity, the results are obtained using a statistical combination of the semi-resolved events with the fully-merged events selected using the criteria in ref. [39]. In addition to improving the search for $\mathrm{X} \rightarrow \mathrm{HH}$, strong constraints are thus obtained for several regions in the $\mathrm{H}$ boson anomalous coupling parameter space, defined by eq. (1.1).

\section{The CMS detector and event reconstruction}

The CMS detector with its coordinate system and the relevant kinematic variables is described in ref. [42]. The central feature of the CMS apparatus is a superconducting solenoid of $6 \mathrm{~m}$ internal diameter, providing a magnetic field of $3.8 \mathrm{~T}$. Within the field volume are silicon pixel and strip trackers, a lead tungstate crystal electromagnetic calorimeter (ECAL), and a brass and scintillator hadron calorimeter (HCAL), each composed of a barrel and two endcap sections. The tracker covers a pseudorapidity $\eta$ range from -2.5 to 2.5 with the ECAL and the HCAL extending up to $|\eta|=3$. Forward calorimeters in the region up to $|\eta|=5$ provide good hermeticity to the detector. Muons are detected in gas-ionization chambers embedded in the steel flux-return yoke outside the solenoid, covering a region of $|\eta|<2.4$.

Events of interest are selected using a two-tiered trigger system [43]. The first level (L1), composed of custom hardware processors, uses information from the calorimeters and muon detectors to select events at a rate of around $100 \mathrm{kHz}$. The second level, known as the high-level trigger (HLT), consists of a farm of processors running a version of the full event reconstruction software optimized for fast processing, and reduces the event rate to around $1 \mathrm{kHz}$ before data storage. Events used in this analysis are selected at the trigger level based on the presence of jets in the detector. The level-1 trigger algorithms reconstruct jets from energy deposits in the calorimeters. The particle-flow (PF) algorithm [44], aims to reconstruct and identify each individual particle in an event. The physics objects reconstructed include jets (clustered with a different algorithm), electrons, muons, photons, and also the missing- $p_{\mathrm{T}}$ vector.

Multiple pp collisions may occur in the same or adjacent LHC bunch crossings (pileup) and contribute to the overall event activity in the detector. The reconstructed vertex with the largest value of summed physics-object $p_{\mathrm{T}}^{2}$ is taken to be the primary pp interaction vertex. The physics objects are the jets, clustered using the jet finding algorithm $[45,46]$ with the tracks assigned tot he vertex as inputs, and the associated missing transverse momentum, taken as the negative vector sum of the $p_{\mathrm{T}}$ of those jets. The other interaction vertices are designated as pileup vertices.

The energy of each electron is determined from a combination of the electron momentum at the primary interaction vertex as determined by the tracker, the energy of the corresponding ECAL cluster, and the energy sum of all bremsstrahlung photons spatially compatible with originating from the electron track. The energy of each muon is obtained 
from the curvature of the corresponding track. The energy of each charged hadron is determined from a combination of its momentum measured in the tracker and the matching ECAL and HCAL energy deposits, corrected for zero-suppression effects and for the response function of the calorimeters to hadronic showers. Finally, the energy of each neutral hadron is obtained from the corresponding corrected ECAL and HCAL energies.

Particles reconstructed by the PF algorithm are clustered into jets with the anti- $k_{\mathrm{T}}$ algorithm [45, 46], using a distance parameter of 0.8 (AK8 jets) or 0.4 (AK4 jets). The jet transverse momentum is determined as the vector sum $p_{\mathrm{T}}$ of all clustered particles. To mitigate the effect of pileup on the AK4 jet momentum, tracks identified as originating from pileup vertices are discarded in the clustering, and an offset correction $[47,48]$ is applied for remaining contributions from neutral particles. Jet energy corrections are derived from simulation to bring the measured response of the jets to that of particle level jets on average. In situ measurements of the momentum balance in events containing either a pair of jets, or a $\mathrm{Z}$ boson or a photon recoiling against a jet, or several jets, are used to account for any residual differences in jet energy scale in data and simulation. Additional selection criteria are applied to each jet to remove jets potentially dominated by anomalous contributions from various subdetector components. After all calibrations, the jet $p_{\mathrm{T}}$ is found from simulation to be within $5-10 \%$ of the true $p_{\mathrm{T}}$ of the clustered particles, over the measured range [48, 49].

For the AK8 jet mass measurement, the "pileup per particle identification" algorithm [50] (PUPPI) is applied to remove pileup effects from the jet. Particles from the PF algorithm, with their PUPPI weights, are clustered into AK8-PUPPI jets which are groomed [51] to remove soft and wide-angle radiation using the soft-drop algorithm [52, 53], using the soft radiation fraction parameter $z=0.1$ and the angular exponent parameter $\beta=0$. Dedicated mass corrections $[39,54]$, derived from simulation and data in a region enriched with $t \overline{\mathrm{t}}$ events containing merged $\mathrm{W} \rightarrow \mathrm{q} \overline{\mathrm{q}}$ decays, are applied to the jet mass in order to remove residual dependence on the jet $p_{\mathrm{T}}$, and to match the jet mass scale and resolution observed in data. The AK8 jet soft-drop mass is assigned by matching the groomed AK8PUPPI jet with the original jet using the criterion $\Delta R$ (AK8 jet, AK8-PUPPI jet) $<0.8$, where $\Delta R \equiv \sqrt{(\Delta \eta)^{2}+(\Delta \phi)^{2}}, \phi$ being the azimuthal angle in radians. The matching efficiency is $100 \%$ in the selected event sample.

\section{Event simulation}

The bulk graviton and radion signal events are simulated at leading order in the mass range $750-3000 \mathrm{GeV}$ with a width of $1 \mathrm{MeV}$ (much smaller than experimental resolution), using the MADGRAPH5_aMC@NLO 2.3.3 [55] event generator. The NNPDF3.0 leading order parton distribution function (PDF) set [56], taken from LHAPDF6 PDF set [57-60], with the four-flavour scheme, is used. The showering and hadronization of partons are simulated with PYTHIA 8.212 [61].

The HERWIG $++2.7 .1[62]$ generator is used as an alternative model, to evaluate the systematic uncertainty associated with the parton shower and hadronization. The tune CUETP8M1-NNPDF2.3LO [63] is used for PyThia 8, while the EE5C tune [64] is used for HERWIG ++ . 


\begin{tabular}{|rrcrcr|}
\hline Shape benchmark & $\kappa_{\lambda}$ & $\kappa_{\mathrm{t}}$ & \multicolumn{1}{c}{$c_{2}$} & \multicolumn{1}{c|}{$c_{\mathrm{g}}$} & $c_{2 \mathrm{~g}}$ \\
\hline 1 & 7.5 & 1.0 & -1.0 & 0.0 & 0.0 \\
2 & 1.0 & 1.0 & 0.5 & -0.8 & 0.6 \\
3 & 1.0 & 1.0 & -1.5 & 0.0 & -0.8 \\
4 & -3.5 & 1.5 & -3.0 & 0.0 & 0.0 \\
5 & 1.0 & 1.0 & 0.0 & 0.8 & -1.0 \\
6 & 2.4 & 1.0 & 0.0 & 0.2 & -0.2 \\
7 & 5.0 & 1.0 & 0.0 & 0.2 & -0.2 \\
8 & 15.0 & 1.0 & 0.0 & -1.0 & 1.0 \\
9 & 1.0 & 1.0 & 1.0 & -0.6 & 0.6 \\
10 & 10.0 & 1.5 & -1.0 & 0.0 & 0.0 \\
11 & 2.4 & 1.0 & 0.0 & 1.0 & -1.0 \\
12 & 15.0 & 1.0 & 1.0 & 0.0 & 0.0 \\
$\mathrm{SM}$ & 1.0 & 1.0 & 0.0 & 0.0 & 0.0 \\
$\kappa_{\lambda}=0$ & 0.0 & 1.0 & 0.0 & 0.0 & 0.0 \\
\hline
\end{tabular}

Table 1. Parameter values of the couplings corresponding to the twelve shape benchmarks, the SM prediction, and the case with vanishing Higgs boson self-interaction, $\kappa_{\lambda}=0$.

Non-resonant HH signals were generated using the effective field theory approach defined in refs. $[4,65]$ and is described by the five parameters given in eq. 1.1: $\kappa_{\lambda}, \kappa_{\mathrm{t}}, c_{2}, c_{\mathrm{g}}$, and $c_{2 \mathrm{~g}}$. The final state kinematic distributions of the $\mathrm{HH}$ pairs depend upon the values of these five parameters. A statistical approach was developed to identify twelve regions of the parameter space, referred to as clusters, with distinct kinematic observables of the $\mathrm{HH}$ system. In particular, models in the same cluster have similar distributions of the di-Higgs boson invariant mass $m_{\mathrm{HH}}$, the transverse momentum of the di-Higgs boson system, and the modulus of the cosine of the polar angle of one Higgs boson with respect to the beam axis, while the distributions of these variables are unique when comparing models from different clusters [66]. For each cluster, a set of representative values of the five parameters is chosen, referred to as the "shape benchmarks". Events are simulated for each of these shape benchmarks, as well as for the SM values of these couplings, and the case where the Higgs boson self-coupling vanishes, i.e. $\kappa_{\lambda}=0$. The values of these benchmark coupling parameters are given in table 1.

The dominant background consists of events comprised uniquely of jets (multijet events) arising from the SM quantum chromodynamics (QCD) interaction, and is modelled entirely from data. The remaining background, consisting mostly of $t \bar{t}+$ jets events, is less than $10 \%$ of the total background, is modelled using POWHEG 2.0 [67-69] and interfaced to PYTHIA 8. The CUETP8M2T4 tune $[70,71]$ is used for generating the $t \bar{t}+$ jets events. The $\mathrm{t} \overline{\mathrm{t}}+\mathrm{jets}$ background rate is estimated using a next-to-next-to-leading order cross section of $832_{-52}^{+46} \mathrm{pb}$ [72], corresponding to the top quark mass of $172.5 \mathrm{GeV}$. A sample of multijet 
events from QCD interactions, simulated at leading order using MADGRAPH5_aMC@NLO and PYTHIA 8, is used to develop and validate the background estimation techniques, prior to being applied to the data.

All generated samples were processed through a GEANT4-based [73, 74] simulation of the CMS detector. The effect of pileup, averaging to 23 at the LHC beam conditions in 2016, is included in the simulations, and the samples are reweighted to match the distribution of the number of pp interactions observed in the data, assuming a total inelastic pp collision cross section of $69.2 \mathrm{mb}[75]$.

\section{Event selection}

Five different HLT triggers were used to collect the semi-resolved events used in this analysis. An event is selected if the scalar sum of the $p_{\mathrm{T}}$ of all AK4 jets in the event $\left(H_{\mathrm{T}}\right)$ is greater than 800 or $900 \mathrm{GeV}$, depending on the LHC beam instantaneous luminosity. Events with $H_{\mathrm{T}} \geq 650 \mathrm{GeV}$, and a pair of jets with invariant mass above $900 \mathrm{GeV}$ and a pseudorapidity separation $|\Delta \eta|<1.5$ are also selected. A third HLT trigger accepts events if the scalar sum of the $p_{\mathrm{T}}$ of all AK8 jets is greater than 650 or $700 \mathrm{GeV}$ and the "trimmed mass" of an AK8 jet is above $50 \mathrm{GeV}$. The jet trimmed mass is obtained after removing remnants of soft radiation with the jet trimming technique [76], using a subjet size parameter of 0.3 and a subjet-to-AK8 jet $p_{\mathrm{T}}$ fraction of 0.1 . Should an event contain an AK8 jet with $p_{\mathrm{T}}>360 \mathrm{GeV}$ and a trimmed mass greater than $30 \mathrm{GeV}$, it is selected by the fourth HLT trigger. Events containing two AK8 jets having $p_{\mathrm{T}}>280$ and $200 \mathrm{GeV}$, with at least one having trimmed mass greater than $30 \mathrm{GeV}$ together with an AK4 jet passing a loose b-tagging criterion, pass the fifth HLT trigger.

Jets in events collected using the logical OR of the above HLT triggers are required to have $|\eta|<2.4$, and $p_{\mathrm{T}}>30 \mathrm{GeV}$ for AK4 jets and $p_{\mathrm{T}}>300 \mathrm{GeV}$ for AK8 jets. One AK8 jet is used to identify a boosted and spatially merged $\mathrm{H} \rightarrow \mathrm{b} \overline{\mathrm{b}}$ decay ( $\mathrm{H}$ jets) while two AK4 jets are used to reconstruct a spatially resolved $\mathrm{H} \rightarrow \mathrm{b} \overline{\mathrm{b}}$ decay.

The first $\mathrm{H}$-tagging criterion requires an AK8 jet to have a soft-drop mass $m_{\mathrm{J}}$ between 105 and $135 \mathrm{GeV}$, consistent with the measured mass of the Higgs boson $m_{\mathrm{H}}=125 \mathrm{GeV}$. This selection corresponds to an efficiency of about $60-70 \%$ for a resonant signal mass $m_{\mathrm{X}}$ in the range $750-3000 \mathrm{GeV}$. The soft-drop jet mass interval was chosen to include a large fraction of the boosted $\mathrm{H} \rightarrow \mathrm{b} \overline{\mathrm{b}}$ signal, while avoiding overlaps with CMS analyses searching for bulk gravitons and radions decaying to boosted $\mathrm{W}$ and $\mathrm{Z}$ bosons [77]. The " $N$-subjettiness" algorithm [78] is used on the AK8-PUPPI jet constituents, to compute the variables $\tau_{\mathrm{N}}$, which quantify the degree to which a jet contains $N$ subjets. A selection on the ratio $\tau_{21} \equiv \tau_{2} / \tau_{1}<0.55$ is required for all AK8 jets to be $\mathrm{H}$ tagged, which has a jet $p_{\mathrm{T}}$-dependent efficiency of $50-70 \%$. The selection criterion on $\tau_{21}$ was optimized for signal sensitivity over the range of $m_{\mathrm{X}}$ values explored.

A jet flavour requirement using a "double-b tagger" algorithm [79] is applied to the AK8 jet as the final H-tagging requirement. The double-b tagger is a multivariate discriminator with an output between -1 and 1 , a higher value indicating a greater probability for the jet to contain $a b \bar{b}$ pair. The double-b tagger exploits the presence of two hadronized 
$\mathrm{b}$ quarks inside the boosted $\mathrm{H} \rightarrow \mathrm{b} \overline{\mathrm{b}}$ decay, and uses variables related to $\mathrm{b}$ hadron lifetime and mass to distinguish $\mathrm{H}$ jets against a background of jets of other flavours. The double-b tagger algorithm also exploits the fact that the directions of the $\mathrm{b}$ hadron momentum are strongly correlated with the axes used to calculate the $N$-subjettiness variables $\tau_{\mathrm{N}}$. An $\mathrm{H}$ jet candidate should have a double-b tagger discriminator greater than 0.8 , which corresponds to an efficiency of $30 \%$ and a misidentification rate of about $1 \%$, as measured in a sample of multijet events. The efficiency of the double-b tagger for simulated jets is corrected to match that in the data, based on efficiency measurements using jets containing pairs of muons, thereby yielding samples enriched in jets from gluons splitting to $b \bar{b}$ pairs. These efficiency corrections are in between 0.92 and 1.02 , for jets in the selected $p_{\mathrm{T}}$ range.

To find a Higgs boson decay into two resolved b quark jets, all AK4 jets in each event are examined for their b tag value using "DeepCSV" algorithm, which is a deep neural network, trained using information from tracks and secondary vertices associated to the jets [79]. The DeepCSV discriminator gives the probability of a jet to have originated from the hadronization of a bottom quark. A selection on the DeepCSV discriminator of AK4 jets is made, corresponding to a $1 \%$ mistag rate for light flavoured jets. The corresponding b-tagging efficiency is about $70 \%$ for b quark jets in the $p_{\mathrm{T}}$ range $80-150 \mathrm{GeV}$, and decreases to about $50 \%$ for $p_{\mathrm{T}} \sim 1000 \mathrm{GeV}$. The $\mathrm{b}$ tagging efficiency in the simulations is corrected to match the one in the data, using measurements of the $\mathrm{b}$ tagging algorithm performance in a sample of muon-tagged jets and $b$ jets from $t \bar{t}+$ jets events, where the correction factor ranges from approximately 0.95 to 1.1 .

To identify events with a resolved $\mathrm{H} \rightarrow \mathrm{b} \overline{\mathrm{b}}$ decay, all pairs of b-tagged AK4 jet are examined, to find events with at least one pair where each AK4 jet is at least $\Delta R>0.8$ away from the leading- $p_{\mathrm{T}}$ AK8 jet and within $\Delta R<1.5$ of each other. If several such pairs are found, the pair of jets, $j_{1}$ and $j_{2}$, that has the highest sum of the AK4 jet DeepCSV discriminator values is selected. The leading- $p_{\mathrm{T}} \mathrm{AK} 8$ jet is then identified as the boosted $\mathrm{H}$ candidate, and the pair of AK4 jets is identified as the resolved $\mathrm{H}$ candidate. If no pairs are found, this process is repeated with the subleading- $p_{\mathrm{T}}$ AK8 jet. If a pair of AK4 jets is identified, then the subleading- $p_{\mathrm{T}} \mathrm{AK} 8$ jet is identified as the boosted $\mathrm{H}$ candidate, and the pair of AK4 jets is identified as the resolved $\mathrm{H}$ candidate. If no pairs are found once again, the event is rejected. The invariant mass of $\mathrm{j}_{1}$ and $\mathrm{j}_{2}, m_{\mathrm{jj}}\left(\mathrm{j}_{1}, \mathrm{j}_{2}\right)$, is required to be within $90-140 \mathrm{GeV}$, forming the resolved $\mathrm{H} \rightarrow \mathrm{b} \overline{\mathrm{b}}$ candidate.

The $t \bar{t}+$ jets background is reduced by reconstructing a $t \rightarrow$ bq $\bar{q}$ system in events with three or more AK4 jets, combining $j_{1}$ and $j_{2}$ with the nearest AK4 jet $\mathrm{j}_{3}$. For the $\mathrm{t} \overline{\mathrm{t}}+$ jets background, the trijet invariant mass $m_{\mathrm{jjj}}\left(\mathrm{j}_{1}, \mathrm{j}_{2}, \mathrm{j}_{3}\right)$ peaks around the top quark mass of $172 \mathrm{GeV}$. Hence, $m_{\mathrm{jjj}}\left(\mathrm{j}_{1}, \mathrm{j}_{2}, \mathrm{j}_{3}\right)$ is required be greater than $200 \mathrm{GeV}$, namely above the top quark mass. Events containing leptons (electrons or muons) with $p_{\mathrm{T}}>20 \mathrm{GeV}$ and $|\eta|<2.4$, and only a small amount of energy in an area around the lepton direction compared to the lepton $p_{\mathrm{T}}$, are rejected, to further suppress $\mathrm{t} \overline{\mathrm{t}}+$ jets and other backgrounds.

A resonant $\mathrm{HH}$ signal results in a small pseudorapidity separation between the two Higgs bosons, while the candidates from the multijet background typically have a larger pseudorapidity separation. Events are therefore categorized according to the pseudorapidity difference between the $\mathrm{H}$ jet and the resolved $\mathrm{H} \rightarrow \mathrm{b} \overline{\mathrm{b}}$ candidate. These two categories 
are defined by $\mid \Delta \eta(\mathrm{H}$ jet, resolved $\mathrm{H} \rightarrow \mathrm{b} \overline{\mathrm{b}}) \mid$ within the interval $0.0-1.0$ or within the interval 1.0-2.0.

To search for resonant and a non-resonant $\mathrm{HH}$ signals, the invariant mass distribution of the boosted and resolved Higgs boson candidate system $\left(m_{\mathrm{Jjj}}\right)$ is examined for an excess of events over the estimated background. The "reduced di-Higgs invariant mass" is defined as $m_{\mathrm{Jjj}, \text { red }} \equiv m_{\mathrm{Jjj}}-\left(m_{\mathrm{J}}-m_{\mathrm{H}}\right)-\left(m_{\mathrm{jj}}\left(\mathrm{j}_{1}, \mathrm{j}_{2}\right)-m_{\mathrm{H}}\right)$. The quantity $m_{\mathrm{Jjj}, \text { red }}$ is used rather than $m_{\mathrm{Jjj}}$ since by subtracting the masses of the reconstructed $\mathrm{H}$ candidates and adding back the exact Higgs boson mass $m_{\mathrm{H}}$, fluctuations from the jet mass resolution are corrected, leading to $8-10 \%$ improvement in the $\mathrm{HH}$ mass resolution. After the full selection, the multijet background is about $90 \%$ of the total background, with the remaining background being $t \bar{t}+$ jets events.

With the above event selection, the trigger criteria reach an efficiency of greater than $99 \%$ for events with $m_{\mathrm{Jjj}, \text { red }} \geq 1100 \mathrm{GeV}$. For lower values of invariant mass (between 750 and $1100 \mathrm{GeV}$ ), the trigger efficiency is between 80 and $99 \%$ for $0 \leq \mid \Delta \eta(\mathrm{H}$ jet, resolved $\mathrm{H} \rightarrow \mathrm{b} \overline{\mathrm{b}}) \mid<1$ and between 60 and $99 \%$ for $1 \leq$ $\mid \Delta \eta(\mathrm{H}$ jet, resolved $\mathrm{H} \rightarrow \mathrm{b} \overline{\mathrm{b}}) \mid \leq 2$. The trigger efficiency for the data is estimated from a multijets sample collected with a control trigger requiring a single AK4 jet with $p_{\mathrm{T}}>260 \mathrm{GeV}$. The trigger efficiency for the simulated samples is corrected using a scale factor to match the observed efficiency for the data. This scale factor depends mildly on $\mid \Delta \eta(\mathrm{H}$ jet, resolved $\mathrm{H} \rightarrow \mathrm{b} \overline{\mathrm{b}}) \mid$, and is hence applied as a function of this variable.

The AK8 jet soft-drop mass distribution, the $N$-subjettiness ratio $\tau_{21}$ distribution, and the double-b tagger discriminator distribution for the backgrounds and simulated signals are shown in figure 1. The DeepCSV discriminator distributions for the two AK4 jets, the dijet invariant mass distribution, and the trijet invariant mass distribution for the backgrounds and simulated samples are shown in figure 2. The selection criteria for the above plots is as follows: AK8 jets with $p_{\mathrm{T}}>300 \mathrm{GeV}$, AK4 jets with $p_{\mathrm{T}}>30 \mathrm{GeV}$, AK8 and AK4 jets with $|\eta|<2.4$, AK8 jet soft-drop mass $>40 \mathrm{GeV}$, AK4 jets DeepCSV discriminator $>0.2219, \Delta R<1.5$ separation between the AK4 jets, and $\Delta R>0.8$ separation between the AK8 jet and each AK4 jet.

The semi-resolved event selection is summarized in table 2, where in addition to these criteria, the events that are used by the fully-merged analysis of ref. [39] are removed, as detailed at the end of this section. The event selection efficiencies for bulk gravitons and radions are given in figure 3 , for different assumed masses in the range $750-2000 \mathrm{GeV}$. At low masses, the efficiency rise is mainly due to the increases in the trigger efficiency and in the efficiency of the requirement on the $|\Delta \eta|$ between the two Higgs boson candidates. The latter efficiency is more important for the radion, which being a spin-0 particle has a wider $|\Delta \eta|$ at low masses than the spin-2 bulk graviton. At high masses, the efficiency drops because more events migrate to the fully-merged regime. The selection efficiencies for the non-resonant signals are between $0.01-2 \%$.

In view of the statistical combination of the semi-resolved and the fully-merged analyses, we briefly describe the search in the fully-merged topology [39]. The analysis in the fully-merged regime uses the same trigger selection and the same selection for the $\mathrm{H}$ jet identification, except for a different requirement on the double-b tagger. These events con- 

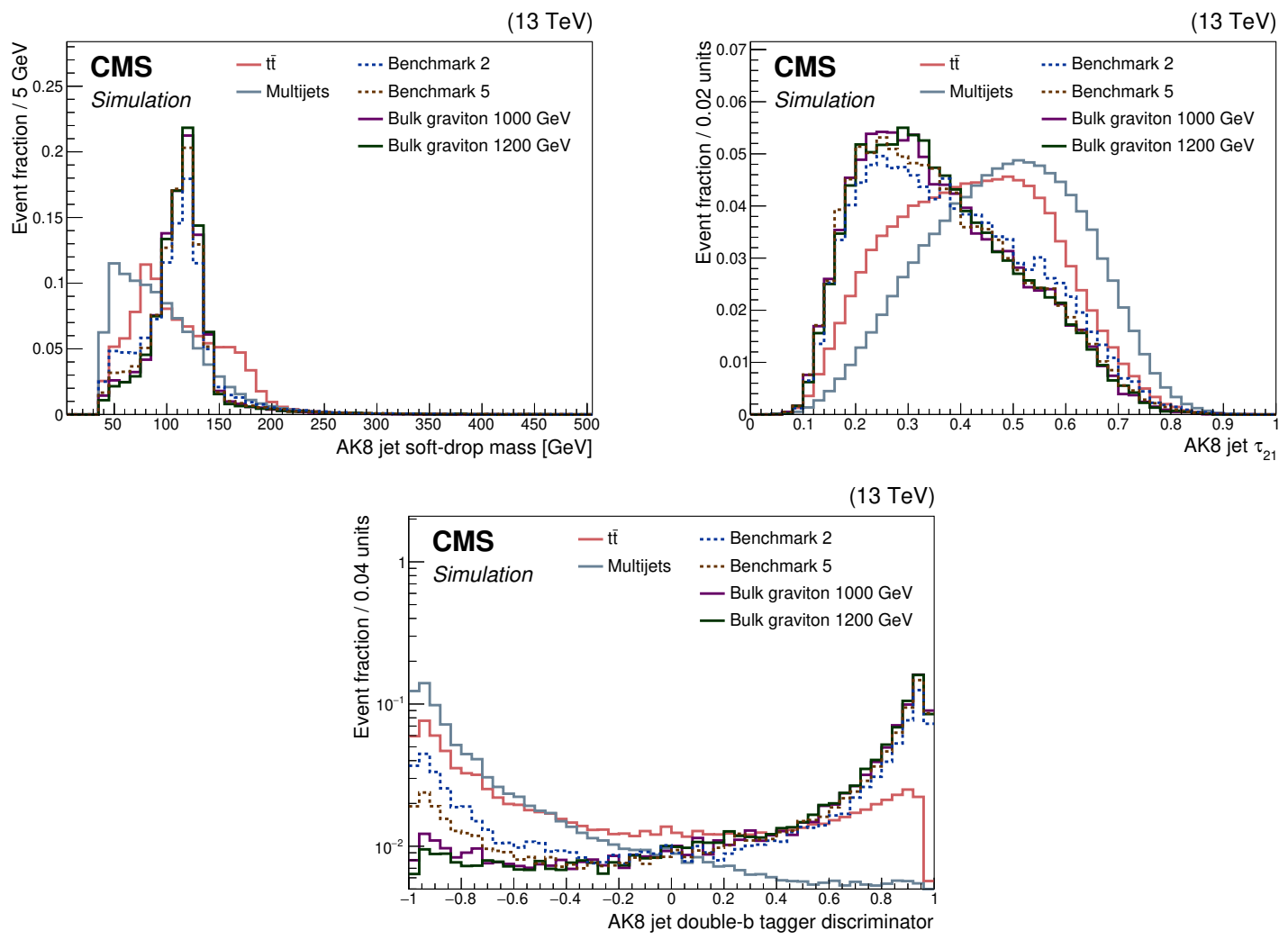

Figure 1. Distributions of the soft-drop mass (upper left), $\tau_{21}$ (upper right), and the doubleb tagger (lower), for AK8 jets in semi-resolved events. The multijet and the t $\bar{t}+$ jets background components are shown separately, along with the simulated signals for bulk gravitons of masses 1000 and $1200 \mathrm{GeV}$ and the non-resonant benchmark models 2 and 5. The distributions are normalized to unity.

tain two $\mathrm{H}$ jets $\mathrm{J}_{1}$ and $\mathrm{J}_{2}$ instead of one. The fully-merged events are classified according to the values of the double-b tagger discriminators of the two $\mathrm{H}$ jets, with both $\mathrm{J}_{1}$ and $\mathrm{J}_{2}$ required to pass a loose double-b tagger discriminator value of $>0.3$. Events are then categorized into those with both $\mathrm{J}_{1}$ and $\mathrm{J}_{2}$ passing a tighter double-b tagger discriminator requirement of $>0.8$, and the rest. The pseudorapidity separation between $\mathrm{J}_{1}$ and $\mathrm{J}_{2}$ is required to be $\left|\Delta \eta\left(\mathrm{J}_{1}, \mathrm{~J}_{2}\right)\right|<1.3$. The reduced di-Higgs invariant mass for fully-merged events is defined as $m_{\mathrm{JJ}, \text { red }}=m_{\mathrm{JJ}}-\left(m_{\mathrm{J}_{1}}-m_{\mathrm{H}}\right)-\left(m_{\mathrm{J}_{2}}-m_{\mathrm{H}}\right)$, where $m_{\mathrm{JJ}}$ is the invariant mass of $\mathrm{J}_{1}$ and $\mathrm{J}_{2}$ and $m_{\mathrm{J}_{1}}$ and $m_{\mathrm{J}_{2}}$ are their soft-drop masses, respectively.

A Higgs boson candidate which passes the boosted AK8 jet selection can also pass the selection for two resolved AK4 jets. In particular, signal samples with higher mass that pass the semi-resolved selection often pass the fully-merged selection because both Higgs candidates are merged, but one candidate still passes the selection for a resolved jet as well. For each signal, the final semi-resolved selection includes anywhere from $23-53 \%$ events that are used by the fully-merged analysis, whether in the signal region or to estimate the QCD multijets background. These events are then removed from the semi-resolved analysis to allow for a combination with the fully-merged analysis. 

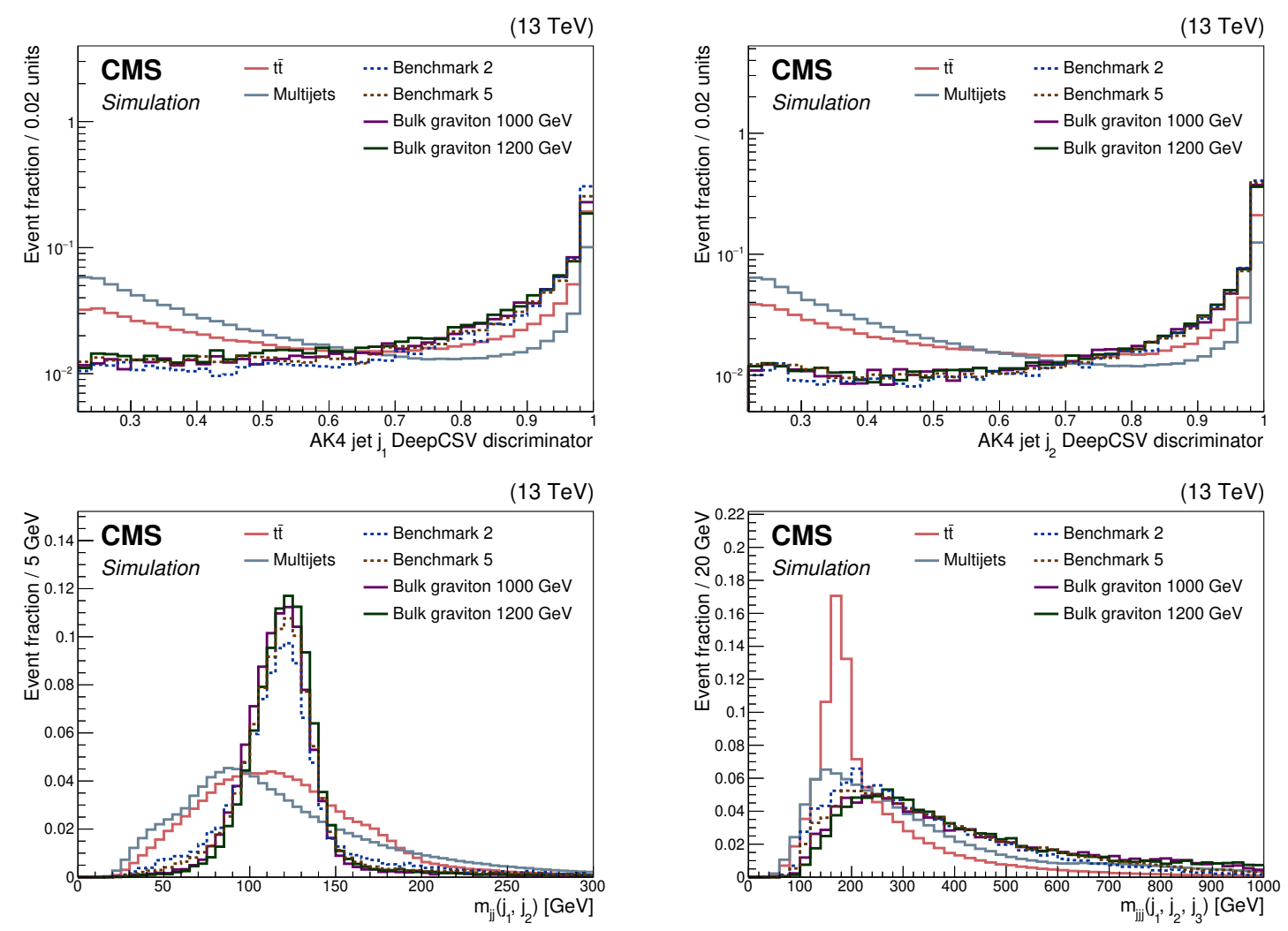

Figure 2. Distributions for AK4 jets of the DeepCSV discriminators for the leading $\mathrm{j}_{1}$ (upper left) and next leading $\mathrm{j}_{2}$ (upper right), the invariant mass of $\mathrm{j}_{1}$ and $\mathrm{j}_{2}, m_{\mathrm{jj}}\left(\mathrm{j}_{1}, \mathrm{j}_{2}\right)$ (lower left), and the invariant mass of $\mathrm{j}_{1}, \mathrm{j}_{2}$, and their nearest $\mathrm{AK} 4$ jet $\mathrm{j}_{3}, m_{\mathrm{Jjj}}\left(\mathrm{j}_{1}, \mathrm{j}_{2}, \mathrm{j}_{3}\right)$ (lower right), in semi-resolved events. The multijet and $t \bar{t}+$ jets background components are shown separately, along with the simulated signals for bulk gravitons of masses 1000 and $1200 \mathrm{GeV}$ and the non-resonant benchmark models 2 and 5 . The distributions are normalized to unity.

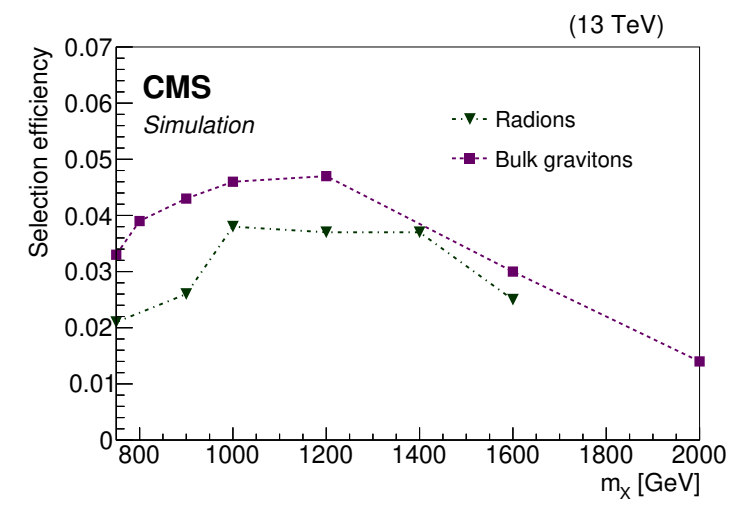

Figure 3. The signal selection efficiencies for the radion and the bulk graviton, for different masses. The events are required to pass the selections given in table 2 as well as to fail the selections of the fully-merged analysis of ref. [39]. 


\begin{tabular}{|c|c|}
\hline Variable & Selection \\
\hline At least 1 AK8 jet $\mathrm{J}$ & $p_{\mathrm{T}}>300 \mathrm{GeV},|\eta|<2.4$ \\
\hline At least 2 AK4 jets $j_{1}$ and $j_{2}$ & $p_{\mathrm{T}}>30 \mathrm{GeV},|\eta|<2.4$ \\
\hline$\Delta R\left(\mathrm{~J}, \mathrm{j}_{\mathrm{i}}\right)$ & $>0.8$ \\
\hline$\Delta R\left(\mathrm{j}_{1}, \mathrm{j}_{2}\right)$ & $<1.5$ \\
\hline$\left|\Delta \eta\left(\mathrm{J}_{,} \mathrm{j}_{1}+\mathrm{j}_{2}\right)\right|$ & $\leq 2$ \\
\hline$m_{\mathrm{Jjj}, \text { red }}$ & $>750 \mathrm{GeV}$ \\
\hline J soft-drop mass & $105-135 \mathrm{GeV}$ \\
\hline $\mathrm{J} \tau_{21}$ & $<0.55$ \\
\hline $\mathrm{J}$ double-b tagger discriminator & $>0.8$ \\
\hline $\mathrm{j}_{1}+\mathrm{j}_{2}$ mass & $90-140 \mathrm{GeV}$ \\
\hline $\mathrm{j}_{1}+\mathrm{j}_{2}+$ (nearest $\mathrm{AK} 4$ jet) mass & $>200 \mathrm{GeV}$ \\
\hline $\mathrm{j}_{1}$ and $\mathrm{j}_{2}$ DeepCSV & $70 \%$ b-tagging eff., $1 \%$ mistag \\
\hline Number of isolated leptons (e or $\mu$ ) & $=0$ \\
\hline
\end{tabular}

Table 2. Summary of the offline selection criteria for semi-resolved $H H \rightarrow b \bar{b} b \bar{b}$ events.

\section{Background estimation}

The multijet background estimation technique for the semi-resolved analysis is the same as that for the fully-merged analysis [39]. A set of signal-free control regions is defined by changing the criteria on the soft-drop mass and the double-b tagger discriminator of the selected AK8 jet from those used for the $\mathrm{H}$ tagging. The selection criteria applied to the AK4 jets forming the resolved $\mathrm{H} \rightarrow \mathrm{b} \overline{\mathrm{b}}$ are the same as those used for the signal regions. If the soft-drop mass is within $60 \mathrm{GeV}$ above or below the $\mathrm{H}$ jet mass window of $105-135 \mathrm{GeV}$, these regions are referred to as the mass sideband regions. These sidebands are separated into regions that pass or fail the double-b tagger tagging requirement.

We define the pass-fail ratio $R_{\mathrm{p} / \mathrm{f}}$ as the ratio of events for which the AK8 jet passes and fails the double-b tagger tagging requirement. The $R_{\mathrm{p} / \mathrm{f}}$ is measured in the soft-drop mass sidebands as a function of soft-drop mass. These values are fit to a quadratic function of the $\mathrm{H}$ jet mass to calculate the $R_{\mathrm{p} / \mathrm{f}}$ in the signal region. The antitag region, defined with the same criteria as the signal region, but with the AK8 jet failing the double-b tagger requirement, is then scaled by the $R_{\mathrm{p} / \mathrm{f}}$ value to estimate the multijets background in the signal region. This is done in bins of soft-drop mass to predict the entire background shape. The dependence of $R_{\mathrm{p} / \mathrm{f}}$ on $m_{\mathrm{Jjj}, \text { red }}$ was found to be negligible, within the measurement uncertainties. Both the shape of the background $m_{\mathrm{Jjj}, \text { red }}$ distribution and its total yield in the signal region is obtained using this method.

Prior to estimating the background, the $t \bar{t}+$ jets contributions derived from Monte Carlo simulation are subtracted from all sideband and signal regions in the data, and then added back in once the multijet background calculation is completed, to estimate 

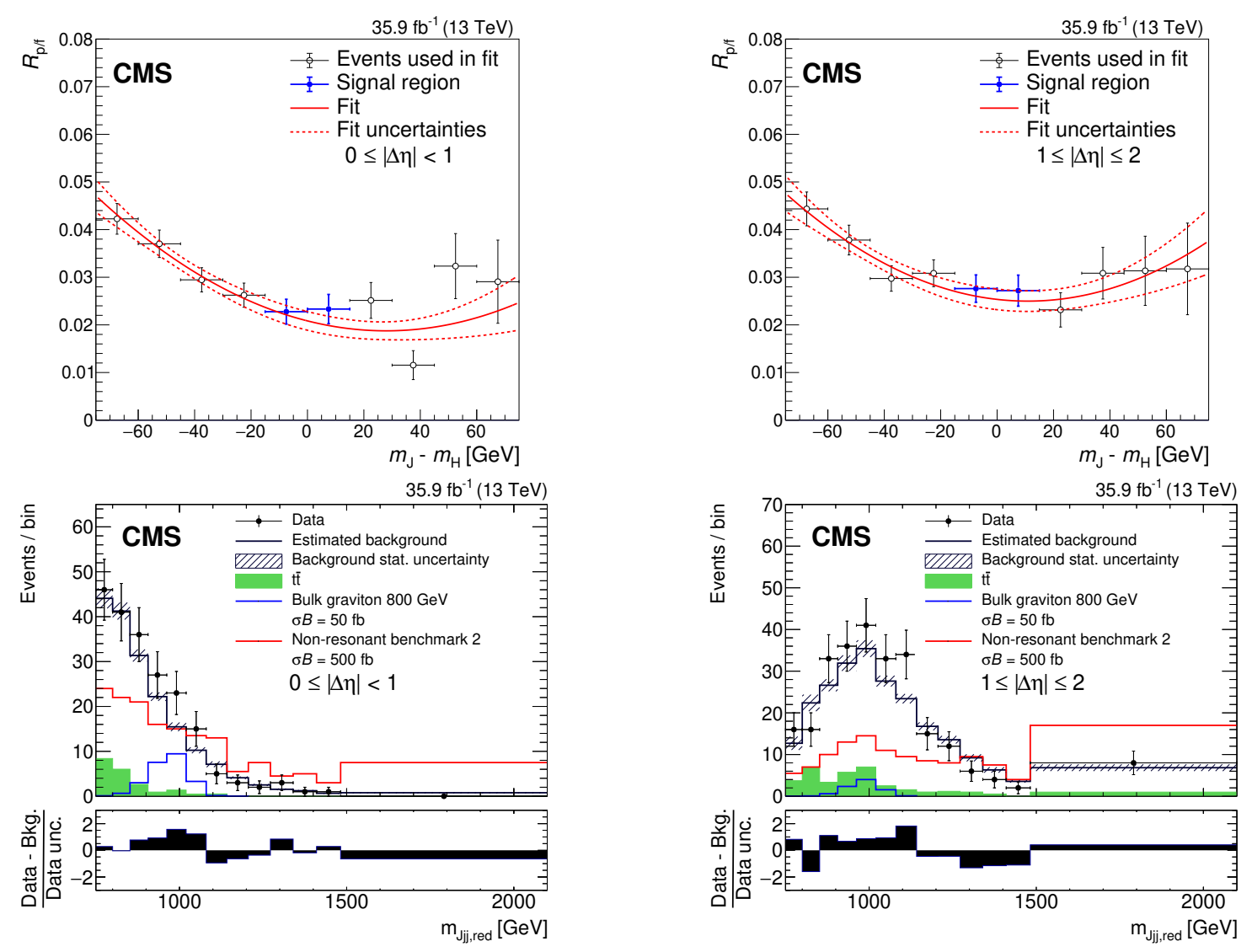

Figure 4. Upper: the double-b tagger pass-fail ratio $R_{\mathrm{p} / \mathrm{f}}$ of the leading- $p_{\mathrm{T}} \mathrm{AK} 8$ jet in semiresolved events as a function of the difference between the soft-drop mass and the Higgs boson mass, $m_{\mathrm{J}}-m_{\mathrm{H}}$. The measured ratio in different bins of $m_{\mathrm{J}}-m_{\mathrm{H}}$ is used in the fit (red solid line), except in the region around $m_{\mathrm{J}}-m_{\mathrm{H}}=0$, which corresponds to the signal region (blue markers). The fitted function is interpolated to obtain $R_{\mathrm{p} / \mathrm{f}}$ in the signal region. Lower: the reduced mass distribution $m_{\mathrm{Jjj}, \text { red }}$ in the data (black markers) with the estimated background represented as the black histogram. The $t \bar{t}+$ jets contribution from simulation is represented in green. The rest of the background is multijets, calculated by applying the $R_{\mathrm{p} / \mathrm{f}}$ to the antitag region. The uncertainty in the total background, before fitting the background model to the data, is depicted using the shaded region. The signal distributions for a bulk graviton with a mass of $800 \mathrm{GeV}$ (blue) and the non-resonant benchmark 2 model (red) are also shown for assumed values of the products of the production cross sections for $\mathrm{HH}$ and the branching fraction to $4 \mathrm{~b}, \sigma \mathcal{B}$. For the left and right figures, the pseudorapidity intervals are $0 \leq|\Delta \eta|<1$ and $1 \leq|\Delta \eta| \leq 2$, respectively. 


\begin{tabular}{|lcc|}
\hline Source & Uncertainty (Semi-resolved) & Uncertainty (Fully-merged) \\
\hline & Signal yield (\%) & \\
Trigger efficiency & $1-15$ & $1-15$ \\
Jet energy scale and resolution & $1-3$ & 1 \\
Jet mass scale and resolution & 2 & 2 \\
H tagging correction factor & $5-20$ & $7-20$ \\
H jet $\tau_{21}$ selection & $+14 /-13$ & $+30 /-26$ \\
b tagging selection & $2-9$ & $2-5$ \\
PDF and scales & $0.1-3$ & $0.1-2$ \\
Pileup modelling & $1-2$ & 2 \\
Luminosity & 2.5 & 2.5 \\
Trijet Invariant Mass & 0.5 & - \\
& Background yield $(\%)$ & - \\
t $\overline{\mathrm{t}}+$ jets cross section & 5 & $2-7$ \\
QCD background $R_{\mathrm{p} / \mathrm{f}}$ fit & $2-10$ & \\
\hline
\end{tabular}

Table 3. Summary of the ranges of systematic uncertainties in the signal and background yields, for both the semi-resolved analysis and for the fully-merged analysis, taken from ref. [39].

the contribution of $t \bar{t}+$ jets to the total background. The fractions of signal events in the sideband regions were found to be negligible as compared with the total numbers of events.

Figure 4 (left) shows the quadratic fit in the AK8 jet soft-drop mass sidebands of the pass-fail ratio $R_{\mathrm{p} / \mathrm{f}}$ as a function of $\mathrm{AK} 8$ jet soft-drop mass, as obtained in the data and in the predicted background shape in the signal region, where overlap with the merged analysis in the signal, sideband, and antitag regions is removed. A $\chi^{2}$ test statistic was used to perform the fit, and the modelling was validated using Monte Carlo simulations and control samples in the data. The functional form was chosen after performing a Fisher F-test [80], which established that, among polynomials, a quadratic form is necessary and sufficient. Other functional forms were tested and the fit results were found to be consistent with that using the quadratic function. The resulting background distributions are compared with the observed data, as shown in figure 4 (right).

\section{$6 \quad$ Systematic uncertainties}

The following sources of systematic uncertainty affect the expected signal and background event yields. None of these lead to a significant change in the signal shape. A complete list of systematic uncertainties is given in table 3 .

The trigger response modelling uncertainties are particularly important for $m_{\mathrm{Jjj}, \text { red }}<$ $1100 \mathrm{GeV}$, where the trigger efficiency drops below 99\%. The trigger efficiency data-tosimulations scale factor has an uncertainty between 1 and $15 \%$, attributable to the control trigger inefficiency and the sample size used. 
The impact of the jet energy scale and resolution uncertainties [54] on the signal yields was estimated to be 1-3\%, depending on the signal mass. The jet mass scale and resolution, as well as the $\tau_{21}$ selection efficiency data-to-simulation scale factors were measured using a sample of boosted $\mathrm{W} \rightarrow \mathrm{q} \overline{\mathrm{q}}^{\prime}$ jets in semileptonic $\mathrm{t} \overline{\mathrm{t}}$ events. The jet mass scale and resolution has a $2 \%$ effect on the signal yields because of a change in the mean of the $\mathrm{H}$ jet mass distribution. A correction factor is applied to account for the difference in the jet shower profile of $\mathrm{W} \rightarrow \mathrm{q} \overline{\mathrm{q}}^{\prime}$ and $\mathrm{H} \rightarrow \mathrm{b} \overline{\mathrm{b}}$ decays, by comparing the ratio of the efficiency of $\mathrm{H}$ and Wjets using the PYTHIA 8 and HERWIG++ shower generators. This uncertainty, the $\mathrm{H}$ tagging correction factor, is in the range $5-20 \%$, depending on the resonance mass $m_{\mathrm{X}}$. The $\tau_{21}$ selection efficiency uncertainty depends on how many $\tau_{21}$ tags are used, two for the fully-merged (26-30\% uncertainty) and one for the semi-resolved analysis (13-15\% uncertainty). This includes an additional uncertainty in the $\tau_{21}$ scale factor, determined using simulations, for jets with $p_{\mathrm{T}}$ higher than those in the $\mathrm{t} \overline{\mathrm{t}}$ events used for the evaluation of this systematic.

Scale factors are used to correct the signal events yields so their double-b tagger and DeepCSV discriminator efficiencies are the same as for data. The double-b tagger and the DeepCSV discriminator scale factors are taken to be $100 \%$ correlated. The associated uncertainty is $2-9 \%$ [79], depending on the double-b tagger and requirement threshold and jet $p_{\mathrm{T}}$, and is propagated to the total uncertainty in the signal yield.

The impact of the theoretical scale uncertainties and PDF uncertainties, the latter derived using the PDF4LHC procedure [60] and the NNPDF3.0 PDF sets, is estimated to be $0.1-3 \%$. These uncertainties affect the product of the signal acceptance and the selection efficiency. The scale and the PDF uncertainties have negligible impact on the signal $m_{\mathrm{Jjj}, \text { red }}$ distributions. Additional systematic uncertainties associated with the pileup modelling (1-2\%, based on a $4.6 \%$ variation on the pp total inelastic cross section) and with the integrated luminosity determination (2.5\%) [81], are applied to the signal yield.

The systematic uncertainty on the trijet invariant mass cut was calculated by comparing the cut efficiency for Pythia and Herwig bulk graviton samples, and is equivalent to a $0.5 \%$ systematic.

The systematic uncertainty applied to the signal is also applied to the $t \bar{t}+$ jets background in the semi-resolved analysis, as appropriate. The total uncertainty in the $t \bar{t}+$ jets background is $11-15 \%$, of which $6 \%$ derives from the uncertainty in the $t \bar{t}+$ jets cross section.

The main source of uncertainty for the multijet background is due to the statistical uncertainty in the fit to the $R_{\mathrm{p} / \mathrm{f}}$ ratio performed in the $\mathrm{H}$ jet mass sidebands. This uncertainty, amounting to $2-10 \%$, is fully correlated between all $m_{\mathrm{Jjj}, \text { red }}$ bins. Additional statistical uncertainties on the background shape and yield in the signal region result from the finite statistics of the multijets samples in the antitag region and are evaluated using the Barlow-Beeston Lite method [82, 83]. These uncertainties are small as compared with the uncertainty on the $R_{\mathrm{p} / \mathrm{f}}$ ratio, and are uncorrelated from bin to bin.

\section{Results}

This analysis extends the search for a resonance $X$ decaying to $\mathrm{HH} \rightarrow \mathrm{b} \overline{\mathrm{b}} \mathrm{b} \overline{\mathrm{b}}$ with two boosted $\mathrm{H}$ jets [39] to cover the semi-resolved topology involving one boosted $\mathrm{H}$ jet and 

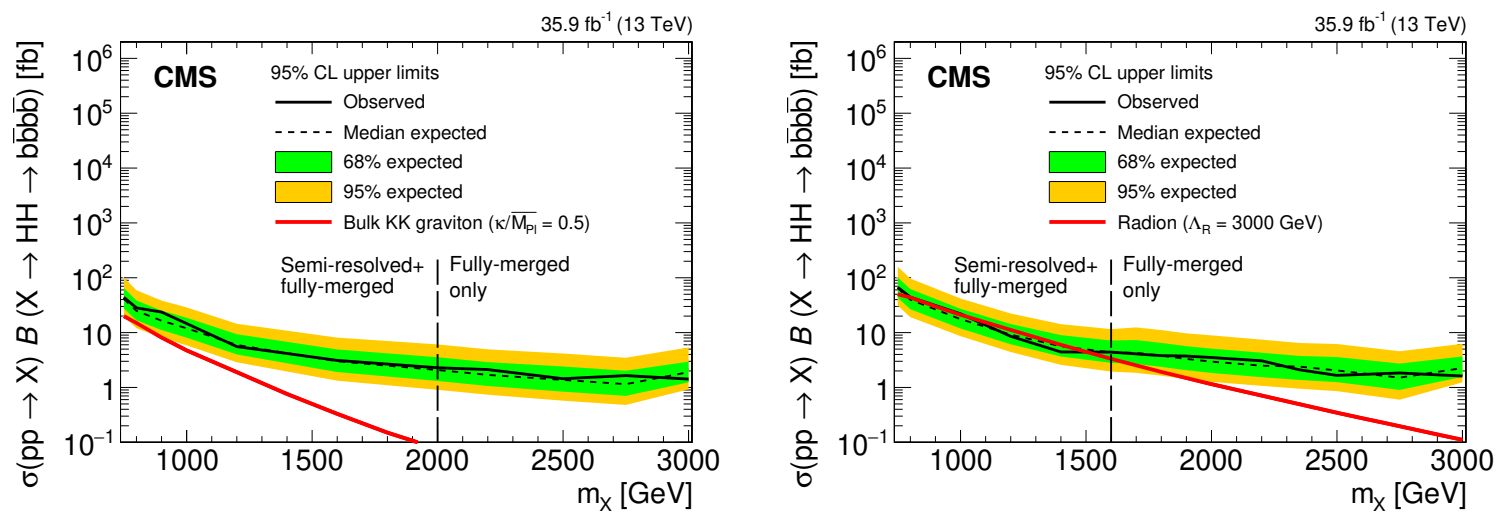

Figure 5. The upper limits for a bulk graviton (left) and radion (right), combining the fully-merged and the semi-resolved analysis (where the events used in the fully-merged analysis are not considered in the semi-resolved analysis). The inner (green) and the outer (yellow) bands indicate the regions containing the 68 and $95 \%$, respectively, of the distribution of limits expected under the backgroundonly hypothesis. The theoretical predictions are shown as the red lines. Results above 2000 (1600) $\mathrm{GeV}$ for the bulk graviton (radion) are taken directly from the fully-merged analysis [39].

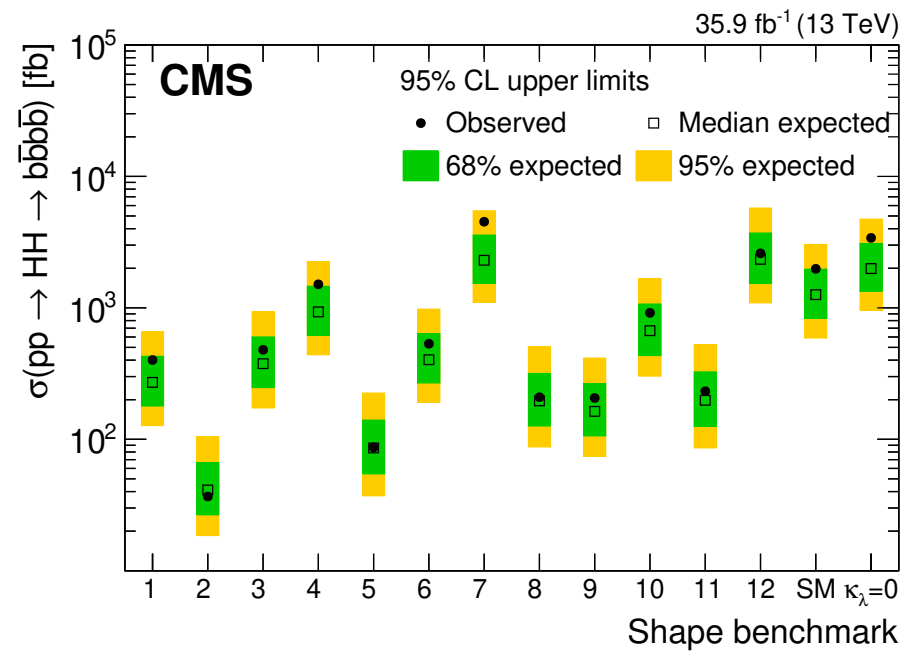

Figure 6. The observed and expected upper limits for non-resonant HH production in the standard model, the model with $\kappa_{\lambda}=0$, and other shape benchmarks (1-12), combining the fully-merged selection and the semi-resolved selection (where the events used in the fully-merged analysis are not considered in the semi-resolved analysis). The inner (green) and the outer (yellow) bands indicate the regions containing the 68 and $95 \%$, respectively, of the distribution of limits expected under the background-only hypothesis.

one resolved $\mathrm{H} \rightarrow \mathrm{b} \overline{\mathrm{b}}$ decay reconstructed using two b jets. An $\mathrm{HH}$ signal would appear as an excess of events over estimated background in the $m_{\mathrm{Jjj}, \text { red }}$ spectra of the different signal event categories, as discussed in section 5 .

The binned $m_{\mathrm{Jjj}, \text { red }}$ distributions of the signal and the background are fitted to the data, as shown in figure 4 (right), and examined for an excess of events above the pre- 
dicted background. The data were found to be consistent with the expected background predictions. Upper limits on the product of the signal cross sections and branching fractions are obtained using the profile likelihood as a test statistic [84]. The systematic uncertainties are treated as nuisance parameters and are profiled in the minimization of the negative of the logarithm of the profile likelihood ratio and the distributions of the likelihood ratio are calculated using the asymptotic approximation [85] of the procedure reported in refs. [86, 87]. Upper limits at $95 \%$ confidence level are set on the product of the production cross section and the branching fractions $\sigma(\mathrm{pp} \rightarrow \mathrm{X}) \mathcal{B}(\mathrm{X} \rightarrow \mathrm{b} \overline{\mathrm{b}} \mathrm{b} \overline{\mathrm{b}})$.

Results are obtained using a statistical combination of the semi-resolved and fullymerged event categories for the bulk graviton having a mass between $750-2000 \mathrm{GeV}$, and a radion with a mass between $750-1600 \mathrm{GeV}$. Above these mass ranges, the inclusion of the semi-resolved events does not appreciably improve the search sensitivity, as evidenced from the expected limit values. The limits on $\sigma(\mathrm{pp} \rightarrow \mathrm{X}) B(\mathrm{X} \rightarrow \mathrm{b} \overline{\mathrm{b}} \mathrm{b} \overline{\mathrm{b}})$ are shown in figure 5, and tabulated in tables 4 and 5 for the bulk graviton and the radion, respectively. The limits for $m_{\mathrm{X}}>2000 \mathrm{GeV}$ for the bulk graviton, and $m_{\mathrm{X}}>1600 \mathrm{GeV}$ for the radion are those from the fully-merged analysis of ref. [39].

For the interpretation of the results, this paper uses the scenario of ref. [88] to describe a KK graviton, where the propagation of SM fields is allowed in the bulk, and follows the characteristics of the SM gauge group, with the right-handed top quark localized near the $\mathrm{TeV}$ brane. The radion is an additional element of WED models that is needed to stabilize the size of the extra dimension $l$. The theoretical cross sections for $\sigma(\mathrm{pp} \rightarrow \mathrm{X}) B(\mathrm{X} \rightarrow \mathrm{b} \overline{\mathrm{b}} \mathrm{b} \overline{\mathrm{b}})$ are calculated using $\kappa / \overline{M_{\mathrm{Pl}}}=0.5$ for the bulk gravitons and $\Lambda_{\mathrm{R}}=3 \mathrm{TeV}$ for the radions, of different masses. For these values of $\kappa / \overline{M_{\mathrm{Pl}}}$ and $\Lambda_{\mathrm{R}}$, the branching fractions $\mathcal{B}(\mathrm{X} \rightarrow \mathrm{b} \overline{\mathrm{b}} \mathrm{b} \overline{\mathrm{b}})$ are 10 and $23 \%$, for the graviton and the radion, respectively [89]. As shown in figure 5 (right), a radion having a mass between 1000 and $1500 \mathrm{GeV}$ is excluded at $95 \%$ confidence level for $\Lambda_{\mathrm{R}}=3 \mathrm{TeV}$.

The improvement in the upper limits on $\sigma(\mathrm{pp} \rightarrow \mathrm{X}) B(\mathrm{X} \rightarrow \mathrm{b} \overline{\mathrm{b}} \mathrm{b} \overline{\mathrm{b}})$ due to the inclusion of the semi-resolved event category between $18 \%$ and $7 \%$, for a bulk graviton in the mass range $750-2000 \mathrm{GeV}$. A much larger improvement - between $55 \%$ and $8 \%$ - is seen for a radion in the mass range $750-1600 \mathrm{GeV}$. This can be attributed to the two pseudorapidity intervals, $|\Delta \eta|<1$ and $1 \leq|\Delta \eta| \leq 2$, utilized in the semi-resolved event selection, with the lower pseudorapidity interval having a better signal to background ratio for a spin-0 radion, because of the angular distribution of its decay products.

In addition, both the fully-merged and the semi-resolved analyses look for non-resonant HH production. The observed and expected upper limits are presented in table 6 for the semi-resolved and the fully-merged signal categories combined, also depicted in figure 6 . The observed and expected limits are respectively, 179 and 114 times the product of the SM cross sections and branching fractions. The new limits are better by about a factor of three for benchmark 2 and a factor of two for benchmark 5, with significant improvements for benchmarks 8, 9, and 11, compared to existing measurements [36, 38]. The increased sensitivity of this analysis to certain benchmarks is due to the higher level of destructive interference among the $\mathrm{HH}$ production processes close to the kinematic threshold, which leads to a corresponding shift of the HH mass spectrum towards higher values. This leads 


\begin{tabular}{|ccccccc|}
\hline $\begin{array}{c}\text { Mass } \\
(\mathrm{GeV})\end{array}$ & $\begin{array}{c}\text { Obs. lim. } \\
(\mathrm{fb})\end{array}$ & $\begin{array}{c}\text { Exp. lim. } \\
(\mathrm{fb})\end{array}$ & $\begin{array}{c}+\operatorname{Exp}(68 \%) \\
(\mathrm{fb})\end{array}$ & $\begin{array}{c}-\operatorname{Exp}(68 \%) \\
(\mathrm{fb})\end{array}$ & $\begin{array}{c}+\operatorname{Exp}(95 \%) \\
(\mathrm{fb})\end{array}$ & $\begin{array}{c}-\operatorname{Exp}(95 \%) \\
(\mathrm{fb})\end{array}$ \\
\hline 750 & 43.9 & 41.0 & 27.4 & 64.8 & 19.6 & 101 \\
800 & 28.2 & 24.9 & 16.7 & 38.5 & 12.1 & 59.2 \\
900 & 23.6 & 16.4 & 11.1 & 25.2 & 8.1 & 38.4 \\
1000 & 14.6 & 11.9 & 8.0 & 18.6 & 5.9 & 28.8 \\
1200 & 5.5 & 5.9 & 3.9 & 9.3 & 2.9 & 14.5 \\
1600 & 3.1 & 3.0 & 1.9 & 4.9 & 1.3 & 8.1 \\
2000 & 2.2 & 2.0 & 1.3 & 3.5 & 0.9 & 6.1 \\
2500 & 1.4 & 1.4 & 0.8 & 2.4 & 0.5 & 4.2 \\
3000 & 1.4 & 1.9 & 1.2 & 3.0 & 0.9 & 5.3 \\
\hline
\end{tabular}

Table 4. The observed and expected upper limits on the products of the cross sections and branching fraction $\sigma(\mathrm{pp} \rightarrow \mathrm{X}) B(\mathrm{X} \rightarrow \mathrm{HH} \rightarrow \mathrm{b} \overline{\mathrm{b}} b \overline{\mathrm{b}})$ for a bulk graviton from the combination of the fully-merged and semi-resolved channels (where the events used in the fully-merged analysis are not considered in the semi-resolved analysis). Results above $2000 \mathrm{GeV}$ are taken directly from the fully-merged analysis [39].

\begin{tabular}{|ccccccc|}
\hline $\begin{array}{c}\text { Mass } \\
(\mathrm{GeV})\end{array}$ & $\begin{array}{c}\text { Obs. lim. } \\
(\mathrm{fb})\end{array}$ & $\begin{array}{c}\text { Exp. lim. } \\
(\mathrm{fb})\end{array}$ & $\begin{array}{c}+\operatorname{Exp}(68 \%) \\
(\mathrm{fb})\end{array}$ & $\begin{array}{c}-\operatorname{Exp}(68 \%) \\
(\mathrm{fb})\end{array}$ & $\begin{array}{c}+\operatorname{Exp}(95 \%) \\
(\mathrm{fb})\end{array}$ & $\begin{array}{c}-\operatorname{Exp}(95 \%) \\
(\mathrm{fb})\end{array}$ \\
\hline 750 & 67.0 & 64.5 & 42.8 & 101 & 30.9 & 158 \\
800 & 44.3 & 39.8 & 26.6 & 62.2 & 19.1 & 96.8 \\
900 & 31.2 & 28.6 & 19.8 & 43.1 & 15.9 & 65.1 \\
1000 & 22.0 & 17.5 & 11.8 & 27.1 & 8.6 & 41.8 \\
1200 & 8.5 & 9.1 & 6.1 & 14.3 & 4.4 & 22.3 \\
1600 & 4.4 & 4.3 & 2.8 & 7.1 & 1.9 & 11.6 \\
2000 & 3.5 & 3.0 & 1.8 & 5.1 & 1.3 & 8.9 \\
2500 & 1.7 & 2.0 & 1.3 & 3.6 & 0.8 & 6.1 \\
3000 & 1.6 & 2.3 & 1.6 & 3.7 & 1.2 & 6.2 \\
\hline
\end{tabular}

Table 5. The observed and expected upper limits on the products of the cross sections and branching fraction $\sigma(\mathrm{pp} \rightarrow \mathrm{X}) B(\mathrm{X} \rightarrow \mathrm{HH} \rightarrow \mathrm{b} \overline{\mathrm{b}} b \overline{\mathrm{b}})$ for a radion from the combination of the fullymerged and semi-resolved channels (where the events used in the fully-merged analysis are not considered in the semi-resolved analysis). Results above $1600 \mathrm{GeV}$ for the radion are taken directly from the fully-merged analysis [39]. 


\begin{tabular}{|ccccccc|}
\hline $\begin{array}{c}\text { Shape } \\
\text { benchmark }\end{array}$ & $\begin{array}{c}\text { Obs. lim. } \\
(\mathrm{fb})\end{array}$ & $\begin{array}{c}\text { Exp. lim. } \\
(\mathrm{fb})\end{array}$ & $\begin{array}{c}+\operatorname{Exp}(68 \%) \\
(\mathrm{fb})\end{array}$ & $\begin{array}{c}-\operatorname{Exp}(68 \%) \\
(\mathrm{fb})\end{array}$ & $\begin{array}{c}+\operatorname{Exp}(95 \%) \\
(\mathrm{fb})\end{array}$ & $\begin{array}{c}-\operatorname{Exp}(95 \%) \\
(\mathrm{fb})\end{array}$ \\
\hline 1 & 401 & 271 & 179 & 428 & 127 & 660 \\
2 & 36.7 & 41.0 & 26.5 & 66.3 & 18.5 & 105 \\
3 & 479 & 376 & 247 & 601 & 173 & 936 \\
4 & 1510 & 932 & 618 & 1460 & 438 & 2240 \\
5 & 86.6 & 85.9 & 54.4 & 140 & 37.0 & 225 \\
6 & 533 & 403 & 268 & 637 & 190 & 978 \\
7 & 4520 & 2300 & 1530 & 3580 & 1100 & 5470 \\
8 & 209 & 196 & 126 & 317 & 87.2 & 504 \\
9 & 206 & 163 & 106 & 264 & 74 & 415 \\
10 & 916 & 670 & 433 & 1070 & 302 & 1660 \\
11 & 232 & 198 & 125 & 326 & 85.9 & 526 \\
12 & 2600 & 2330 & 1530 & 3700 & 1090 & 5750 \\
SM & 1980 & 1260 & 833 & 1970 & 589 & 3030 \\
$\kappa_{\lambda}=0$ & 3404 & 1989 & 3092 & 1334 & 4732 & 960 \\
\hline
\end{tabular}

Table 6. The observed and expected upper limits on the cross section $\sigma(\mathrm{pp} \rightarrow \mathrm{HH} \rightarrow \mathrm{b} \bar{b} b \bar{b})$ for the non-resonant shape benchmark models (1-12), the SM, and the $\kappa_{\lambda}=0 \mathrm{HH}$ productions, combining fully-merged and semi-resolved channels (where the events used in the fully-merged analysis are not considered in the semi-resolved analysis).

to, on average, a higher $p_{\mathrm{T}}$ of the Higgs bosons, and hence in the sensitivity of this analysis, which identifies Higgs bosons using boosted techniques.

\section{Summary}

A search is presented for the pair production of standard model Higgs bosons (HH), both decaying to a bottom quark-antiquark pair $(\mathrm{b} \overline{\mathrm{b}})$, using data from proton-proton collisions at a centre-of-mass energy of $13 \mathrm{TeV}$ and corresponding to an integrated luminosity of $35.9 \mathrm{fb}^{-1}$. The search is conducted in the region of phase space where at least one of the Higgs bosons has a large Lorentz boost, so that the $\mathrm{H} \rightarrow \mathrm{b} \overline{\mathrm{b}}$ decay products are collimated to form a single jet, an $\mathrm{H}$ jet. The search combines events with one $\mathrm{H}$ jet plus two $\mathrm{b}$ jets with events having two $\mathrm{H}$ jets, thus adding sensitivity to the previous analysis [39].

The results of the search are compared with predictions for the resonant production of a narrow Kaluza-Klein bulk graviton and a narrow radion in warped extradimensional models. The search is also sensitive to several beyond standard model non-resonant $\mathrm{HH}$ production scenarios. Such cases may arise either when an off-shell massive resonance produced in proton-proton collisions decays to $\mathrm{HH}$, or through beyond standard model effects in the Higgs boson coupling parameters. The results are interpreted in terms of upper limits on the product of the cross section for the respective signal processes and the branching fraction to $\mathrm{HH} \rightarrow \mathrm{b} \overline{\mathrm{b}} \mathrm{b} \overline{\mathrm{b}}$, at $95 \%$ confidence level. 
The upper limits range from 43.9 to $1.4 \mathrm{fb}$ for the bulk graviton and from 67 to $1.6 \mathrm{fb}$ for the radion for the mass range $750-3000 \mathrm{GeV}$. Depending on the mass of the resonance, these limits improve upon the results of ref. [39] by up to $18 \%$ for the bulk graviton and up to $55 \%$ for the radion.

The non-resonant production of Higgs boson pairs is modelled using an effective Lagrangian with five coupling parameters. The upper limit corresponding to the standard model values of the coupling parameters is placed at $1980 \mathrm{fb}$, which is 179 times the prediction. In addition, upper limits in the range of 4520 to $36.7 \mathrm{fb}$ are set on twelve shape benchmarks, i.e. representative sets of the five coupling parameters [66]. These are the first limits on non-resonant Higgs boson pair-production signals using boosted topologies, and are the most stringent limits to date for the shape benchmarks $2,5,8,9$, and 11 .

\section{Acknowledgments}

We congratulate our colleagues in the CERN accelerator departments for the excellent performance of the LHC and thank the technical and administrative staffs at CERN and at other CMS institutes for their contributions to the success of the CMS effort. In addition, we gratefully acknowledge the computing centres and personnel of the Worldwide LHC Computing Grid for delivering so effectively the computing infrastructure essential to our analyses. Finally, we acknowledge the enduring support for the construction and operation of the LHC and the CMS detector provided by the following funding agencies: BMBWF and FWF (Austria); FNRS and FWO (Belgium); CNPq, CAPES, FAPERJ, FAPERGS, and FAPESP (Brazil); MES (Bulgaria); CERN; CAS, MoST, and NSFC (China); COLCIENCIAS (Colombia); MSES and CSF (Croatia); RPF (Cyprus); SENESCYT (Ecuador); MoER, ERC IUT, and ERDF (Estonia); Academy of Finland, MEC, and HIP (Finland); CEA and CNRS/IN2P3 (France); BMBF, DFG, and HGF (Germany); GSRT (Greece); NKFIA (Hungary); DAE and DST (India); IPM (Iran); SFI (Ireland); INFN (Italy); MSIP and NRF (Republic of Korea); MES (Latvia); LAS (Lithuania); MOE and UM (Malaysia); BUAP, CINVESTAV, CONACYT, LNS, SEP, and UASLP-FAI (Mexico); MOS (Montenegro); MBIE (New Zealand); PAEC (Pakistan); MSHE and NSC (Poland); FCT (Portugal); JINR (Dubna); MON, RosAtom, RAS, RFBR, and NRC KI (Russia); MESTD (Serbia); SEIDI, CPAN, PCTI, and FEDER (Spain); MOSTR (Sri Lanka); Swiss Funding Agencies (Switzerland); MST (Taipei); ThEPCenter, IPST, STAR, and NSTDA (Thailand); TUBITAK and TAEK (Turkey); NASU and SFFR (Ukraine); STFC (United Kingdom); DOE and NSF (U.S.A.).

Individuals have received support from the Marie-Curie programme and the European Research Council and Horizon 2020 Grant, contract No. 675440 (European Union); the Leventis Foundation; the A. P. Sloan Foundation; the Alexander von Humboldt Foundation; the Belgian Federal Science Policy Office; the Fonds pour la Formation à la Recherche dans l'Industrie et dans l'Agriculture (FRIA-Belgium); the Agentschap voor Innovatie door Wetenschap en Technologie (IWT-Belgium); the F.R.S.-FNRS and FWO (Belgium) under the "Excellence of Science — EOS" — be.h project n. 30820817; the Ministry of Education, Youth and Sports (MEYS) of the Czech Republic; the Lendület ("Momentum") Pro- 
gramme and the János Bolyai Research Scholarship of the Hungarian Academy of Sciences, the New National Excellence Program ÚNKP, the NKFIA research grants 123842, 123959, 124845, 124850 and 125105 (Hungary); the Council of Science and Industrial Research, India; the HOMING PLUS programme of the Foundation for Polish Science, cofinanced from European Union, Regional Development Fund, the Mobility Plus programme of the Ministry of Science and Higher Education, the National Science Center (Poland), contracts Harmonia 2014/14/M/ST2/00428, Opus 2014/13/B/ST2/02543, 2014/15/B/ST2/03998, and 2015/19/B/ST2/02861, Sonata-bis 2012/07/E/ST2/01406; the National Priorities Research Program by Qatar National Research Fund; the Programa Estatal de Fomento de la Investigación Científica y Técnica de Excelencia María de Maeztu, grant MDM-2015-0509 and the Programa Severo Ochoa del Principado de Asturias; the Thalis and Aristeia programmes cofinanced by EU-ESF and the Greek NSRF; the Rachadapisek Sompot Fund for Postdoctoral Fellowship, Chulalongkorn University and the Chulalongkorn Academic into Its 2nd Century Project Advancement Project (Thailand); the Welch Foundation, contract C-1845; and the Weston Havens Foundation (U.S.A.).

Open Access. This article is distributed under the terms of the Creative Commons Attribution License (CC-BY 4.0), which permits any use, distribution and reproduction in any medium, provided the original author(s) and source are credited.

\section{References}

[1] ATLAS collaboration, Observation of a new particle in the search for the Standard Model Higgs boson with the ATLAS detector at the LHC, Phys. Lett. B 716 (2012) 1 [arXiv:1207.7214] [INSPIRE].

[2] CMS collaboration, Observation of a new boson at a mass of $125 \mathrm{GeV}$ with the CMS experiment at the LHC, Phys. Lett. B 716 (2012) 30 [arXiv:1207.7235] [INSPIRE].

[3] CMS collaboration, Observation of a new boson with mass near $125 \mathrm{GeV}$ in pp collisions at $\sqrt{s}=7$ and $8 \mathrm{TeV}$, JHEP 06 (2013) 081 [arXiv:1303.4571] [INSPIRE].

[4] LHC Higgs Cross Section Working Group collaboration, Handbook of LHC Higgs cross sections: 4. Deciphering the nature of the Higgs sector, CERN-2017-002-M, CERN, Geneva, Switzerland (2016) [arXiv: 1610.07922] [INSPIRE].

[5] D. de Florian and J. Mazzitelli, Higgs boson pair production at next-to-next-to-leading order in QCD, Phys. Rev. Lett. 111 (2013) 201801 [arXiv:1309.6594] [INSPIRE].

[6] J. Baglio, A. Djouadi, R. Gröber, M.M. Mühlleitner, J. Quevillon and M. Spira, The measurement of the Higgs self-coupling at the LHC: theoretical status, JHEP 04 (2013) 151 [arXiv: 1212.5581] [INSPIRE].

[7] L. Randall and R. Sundrum, A large mass hierarchy from a small extra dimension, Phys. Rev. Lett. 83 (1999) 3370 [hep-ph/9905221] [INSPIRE].

[8] R. Grober and M. Muhlleitner, Composite Higgs boson pair production at the LHC, JHEP 06 (2011) 020 [arXiv: 1012.1562] [INSPIRE].

[9] W.D. Goldberger and M.B. Wise, Modulus stabilization with bulk fields, Phys. Rev. Lett. 83 (1999) 4922 [hep-ph/9907447] [INSPIRE]. 
[10] O. DeWolfe, D.Z. Freedman, S.S. Gubser and A. Karch, Modeling the fifth-dimension with scalars and gravity, Phys. Rev. D 62 (2000) 046008 [hep-th/9909134] [INSPIRE].

[11] C. Csáki, M. Graesser, L. Randall and J. Terning, Cosmology of brane models with radion stabilization, Phys. Rev. D 62 (2000) 045015 [hep-ph/9911406] [inSPIRE].

[12] H. Davoudiasl, J.L. Hewett and T.G. Rizzo, Phenomenology of the Randall-Sundrum gauge hierarchy model, Phys. Rev. Lett. 84 (2000) 2080 [hep-ph/9909255] [INSPIRE].

[13] C. Csáki, M.L. Graesser and G.D. Kribs, Radion dynamics and electroweak physics, Phys. Rev. D 63 (2001) 065002 [hep-th/0008151] [INSPIRE].

[14] K. Agashe, H. Davoudiasl, G. Perez and A. Soni, Warped gravitons at the LHC and beyond, Phys. Rev. D 76 (2007) 036006 [hep-ph/0701186] [InSPIRE].

[15] G.F. Giudice, R. Rattazzi and J.D. Wells, Graviscalars from higher dimensional metrics and curvature Higgs mixing, Nucl. Phys. B 595 (2001) 250 [hep-ph/0002178] [INSPIRE].

[16] S. Dawson, A. Ismail and I. Low, What's in the loop? The anatomy of double Higgs production, Phys. Rev. D 91 (2015) 115008 [arXiv: 1504.05596] [INSPIRE].

[17] ATLAS and CMS collaborations, Combined measurement of the Higgs boson mass in pp collisions at $\sqrt{s}=7$ and $8 \mathrm{TeV}$ with the ATLAS and CMS experiments, Phys. Rev. Lett. 114 (2015) 191803 [arXiv: 1503.07589] [INSPIRE].

[18] CMS collaboration, Measurements of properties of the Higgs boson decaying into the four-lepton final state in pp collisions at $\sqrt{s}=13 \mathrm{TeV}$, JHEP 11 (2017) 047 [arXiv: 1706.09936] [INSPIRE].

[19] G.F. Giudice, C. Grojean, A. Pomarol and R. Rattazzi, The strongly-interacting light Higgs, JHEP 06 (2007) 045 [hep-ph/0703164] [INSPIRE].

[20] CMS collaboration, Precise determination of the mass of the Higgs boson and tests of compatibility of its couplings with the Standard Model predictions using proton collisions at 7 and $8 \mathrm{TeV}$, Eur. Phys. J. C 75 (2015) 212 [arXiv:1412.8662] [INSPIRE].

[21] ATLAS collaboration, Measurements of the Higgs boson production and decay rates and coupling strengths using pp collision data at $\sqrt{s}=7$ and $8 \mathrm{TeV}$ in the ATLAS experiment, Eur. Phys. J. C 76 (2016) 6 [arXiv: 1507.04548] [InSPIRE].

[22] ATLAS collaboration, Search for Higgs boson pair production in the $\gamma \gamma b \bar{b}$ final state using pp collision data at $\sqrt{s}=8 \mathrm{TeV}$ from the ATLAS detector, Phys. Rev. Lett. 114 (2015) 081802 [arXiv: 1406.5053] [INSPIRE].

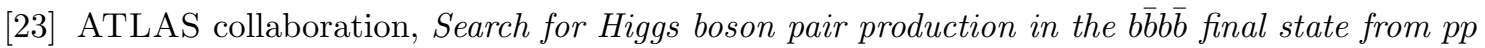
collisions at $\sqrt{s}=8 \mathrm{TeV}$ with the ATLAS detector, Eur. Phys. J. C $7 \mathbf{5}$ (2015) 412 [arXiv: 1506.00285] [INSPIRE].

[24] ATLAS collaboration, Searches for Higgs boson pair production in the $h h \rightarrow b b \tau \tau, \gamma \gamma W W^{*}$, $\gamma \gamma b b$, bbbb channels with the ATLAS detector, Phys. Rev. D 92 (2015) 092004 [arXiv: 1509.04670] [INSPIRE].

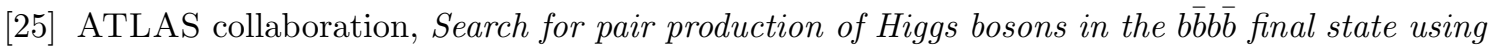
proton-proton collisions at $\sqrt{s}=13 \mathrm{TeV}$ with the ATLAS detector, Phys. Rev. D 94 (2016) 052002 [arXiv: 1606. 04782] [INSPIRE]. 
[26] ATLAS collaboration, Search for pair production of Higgs bosons in the b $\bar{b} b \bar{b}$ final state using proton-proton collisions at $\sqrt{s}=13 \mathrm{TeV}$ with the ATLAS detector, arXiv:1804.06174 [INSPIRE].

[27] ATLAS collaboration, Search for resonant and nonresonant Higgs boson pair production in the $b \bar{b} \tau^{+} \tau^{-}$decay channel in pp collisions at $\sqrt{s}=13 \mathrm{TeV}$ with the ATLAS detector, Phys. Rev. Lett. 121 (2018) 191801 [arXiv:1808.00336] [INSPIRE].

[28] ATLAS collaboration, Search for Higgs boson pair production in the $\gamma \gamma W W^{*}$ channel using pp collision data recorded at $\sqrt{s}=13 \mathrm{TeV}$ with the ATLAS detector, Eur. Phys. J. C 78 (2018) 1007 [arXiv: 1807.08567] [INSPIRE].

[29] ATLAS collaboration, Search for Higgs boson pair production in the $\gamma \gamma b \bar{b}$ final state with $13 \mathrm{TeV}$ pp collision data collected by the ATLAS experiment, JHEP 11 (2018) 040 [arXiv: 1807.04873] [INSPIRE].

[30] CMS collaboration, Searches for heavy Higgs bosons in two-Higgs-doublet models and for $t \rightarrow$ ch decay using multilepton and diphoton final states in pp collisions at 8 TeV, Phys. Rev. D 90 (2014) 112013 [arXiv:1410.2751] [INSPIRE].

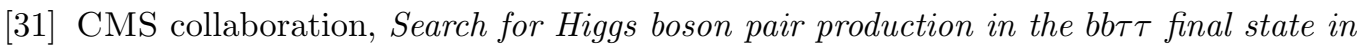
proton-proton collisions at $\sqrt{s}=8$ TeV, Phys. Rev. D 96 (2017) 072004 [arXiv:1707.00350] [INSPIRE].

[32] CMS collaboration, Search for resonant pair production of Higgs bosons decaying to two bottom quark-antiquark pairs in proton-proton collisions at 8 TeV, Phys. Lett. B 749 (2015) 560 [arXiv: 1503.04114] [INSPIRE].

[33] CMS collaboration, Searches for a heavy scalar boson $H$ decaying to a pair of 125 GeV Higgs bosons hh or for a heavy pseudoscalar boson $A$ decaying to $Z h$, in the final states with $h \rightarrow \tau \tau$, Phys. Lett. B 755 (2016) 217 [arXiv:1510.01181] [INSPIRE].

[34] CMS collaboration, Search for two Higgs bosons in final states containing two photons and two bottom quarks in proton-proton collisions at 8 TeV, Phys. Rev. D 94 (2016) 052012 [arXiv: 1603.06896] [INSPIRE].

[35] CMS collaboration, Search for heavy resonances decaying to two Higgs bosons in final states containing four b quarks, Eur. Phys. J. C 76 (2016) 371 [arXiv:1602.08762] [INSPIRE].

[36] CMS collaboration, Search for Higgs boson pair production in events with two bottom quarks and two tau leptons in proton-proton collisions at $\sqrt{s}=13$ TeV, Phys. Lett. B 778 (2018) 101 [arXiv: 1707.02909] [INSPIRE].

[37] CMS collaboration, Search for resonant and nonresonant Higgs boson pair production in the $b \bar{b} \ell \nu \ell \nu$ final state in proton-proton collisions at $\sqrt{s}=13 \mathrm{TeV}$, JHEP 01 (2018) 054 [arXiv: 1708.04188] [INSPIRE].

[38] CMS collaboration, Search for Higgs boson pair production in the $\gamma \gamma b \bar{b}$ final state in $p p$ collisions at $\sqrt{s}=13 \mathrm{TeV}$, Phys. Lett. B 788 (2019) 7 [arXiv:1806.00408] [inSPIRE].

[39] CMS collaboration, Search for a massive resonance decaying to a pair of Higgs bosons in the four b quark final state in proton-proton collisions at $\sqrt{s}=13$ TeV, Phys. Lett. B 781 (2018) 244 [arXiv: 1710.04960] [INSPIRE].

[40] CMS collaboration, Search for resonant pair production of Higgs bosons decaying to bottom quark-antiquark pairs in proton-proton collisions at 13 TeV, JHEP 08 (2018) 152 [arXiv: 1806.03548] [INSPIRE]. 
[41] M. Gouzevitch, A. Oliveira, J. Rojo, R. Rosenfeld, G.P. Salam and V. Sanz, Scale-invariant resonance tagging in multijet events and new physics in Higgs pair production, JHEP $\mathbf{0 7}$ (2013) 148 [arXiv:1303.6636] [INSPIRE].

[42] CMS collaboration, The CMS experiment at the CERN LHC, 2008 JINST 3 S08004 [INSPIRE].

[43] CMS collaboration, The CMS trigger system, 2017 JINST 12 P01020 [arXiv:1609.02366] [INSPIRE].

[44] CMS collaboration, Particle-flow reconstruction and global event description with the CMS detector, 2017 JINST 12 P10003 [arXiv:1706.04965] [INSPIRE].

[45] M. Cacciari, G.P. Salam and G. Soyez, The anti- $k_{t}$ jet clustering algorithm, JHEP 04 (2008) 063 [arXiv: 0802.1189] [INSPIRE].

[46] M. Cacciari, G.P. Salam and G. Soyez, FastJet user manual, Eur. Phys. J. C 72 (2012) 1896 [arXiv:1111.6097] [INSPIRE].

[47] M. Cacciari and G.P. Salam, Pileup subtraction using jet areas, Phys. Lett. B 659 (2008) 119 [arXiv: 0707.1378] [INSPIRE].

[48] CMS collaboration, Jet energy scale and resolution in the CMS experiment in pp collisions at $8 \mathrm{TeV}, 2017$ JINST 12 P02014 [arXiv: 1607.03663] [INSPIRE].

[49] CMS collaboration, Jet energy scale and resolution performances with $13 \mathrm{TeV}$ data, CMS-DP-2016-020, CERN, Geneva, Switzerland, (2016).

[50] D. Bertolini, P. Harris, M. Low and N. Tran, Pileup per particle identification, JHEP 10 (2014) 059 [arXiv: 1407.6013] [INSPIRE].

[51] G.P. Salam, Towards jetography, Eur. Phys. J. C 67 (2010) 637 [arXiv:0906.1833] [INSPIRE].

[52] M. Dasgupta, A. Fregoso, S. Marzani and G.P. Salam, Towards an understanding of jet substructure, JHEP 09 (2013) 029 [arXiv:1307.0007] [INSPIRE].

[53] A.J. Larkoski, S. Marzani, G. Soyez and J. Thaler, Soft drop, JHEP 05 (2014) 146 [arXiv: 1402.2657] [INSPIRE].

[54] CMS collaboration, Jet algorithms performance in $13 \mathrm{TeV}$ data, CMS-PAS-JME-16-003, CERN, Geneva, Switzerland, (2017).

[55] J. Alwall et al., The automated computation of tree-level and next-to-leading order differential cross sections and their matching to parton shower simulations, JHEP 07 (2014) 079 [arXiv: 1405.0301] [INSPIRE].

[56] NNPDF collaboration, Parton distributions for the LHC run II, JHEP 04 (2015) 040 [arXiv: 1410.8849] [INSPIRE].

[57] L.A. Harland-Lang, A.D. Martin, P. Motylinski and R.S. Thorne, Parton distributions in the LHC era: MMHT 2014 PDFs, Eur. Phys. J. C 75 (2015) 204 [arXiv:1412.3989] [InSPIRE].

[58] A. Buckley et al., LHAPDF6: parton density access in the LHC precision era, Eur. Phys. J. C 75 (2015) 132 [arXiv: 1412.7420] [INSPIRE].

[59] S. Carrazza, J.I. Latorre, J. Rojo and G. Watt, A compression algorithm for the combination of PDF sets, Eur. Phys. J. C 75 (2015) 474 [arXiv: 1504.06469] [inSPIRE]. 
[60] J. Butterworth et al., PDF4LHC recommendations for LHC run II, J. Phys. G 43 (2016) 023001 [arXiv: 1510.03865] [INSPIRE].

[61] T. Sjöstrand et al., An introduction to PYTHIA 8.2, Comput. Phys. Commun. 191 (2015) 159 [arXiv:1410.3012] [INSPIRE].

[62] M. Bähr et al., HERWIG++ physics and manual, Eur. Phys. J. C 58 (2008) 639 [arXiv: 0803.0883] [INSPIRE].

[63] CMS collaboration, Event generator tunes obtained from underlying event and multiparton scattering measurements, Eur. Phys. J. C 76 (2016) 155 [arXiv:1512.00815] [INSPIRE].

[64] S. Gieseke, C. Rohr and A. Siodmok, Colour reconnections in HERWIG++, Eur. Phys. J. C 72 (2012) 2225 [arXiv: 1206.0041] [INSPIRE].

[65] A. Carvalho et al., Analytical parametrization and shape classification of anomalous $H H$ production in the EFT approach, arXiv:1608.06578 [INSPIRE].

[66] A. Carvalho, M. Dall'Osso, T. Dorigo, F. Goertz, C.A. Gottardo and M. Tosi, Higgs pair production: choosing benchmarks with cluster analysis, JHEP 04 (2016) 126 [arXiv: 1507.02245] [INSPIRE].

[67] S. Frixione, P. Nason and G. Ridolfi, A positive-weight next-to-leading-order Monte Carlo for heavy flavour hadroproduction, JHEP 09 (2007) 126 [arXiv:0707.3088] [INSPIRE].

[68] S. Frixione, P. Nason and C. Oleari, Matching NLO QCD computations with parton shower simulations: the POWHEG method, JHEP 11 (2007) 070 [arXiv:0709.2092] [INSPIRE].

[69] S. Alioli, P. Nason, C. Oleari and E. Re, A general framework for implementing NLO calculations in shower Monte Carlo programs: the POWHEG BOX, JHEP 06 (2010) 043 [arXiv: 1002.2581] [INSPIRE].

[70] P. Skands, S. Carrazza and J. Rojo, Tuning PYTHIA 8.1: the Monash 2013 tune, Eur. Phys. J. C 74 (2014) 3024 [arXiv: 1404.5630] [INSPIRE].

[71] CMS collaboration, Measurement of the top quark mass with lepton+jets final states using pp collisions at $\sqrt{s}=13 \mathrm{TeV}$, Eur. Phys. J. C 78 (2018) 891 [arXiv:1805.01428] [InSPIRE].

[72] M. Czakon and A. Mitov, Top++: a program for the calculation of the top-pair cross-section at hadron colliders, Comput. Phys. Commun. 185 (2014) 2930 [arXiv:1112.5675] [INSPIRE].

[73] GEANT4 collaboration, GEANT4: a simulation toolkit, Nucl. Instrum. Meth. A 506 (2003) 250 [INSPIRE].

[74] J. Allison et al., GEANT4 developments and applications, IEEE Trans. Nucl. Sci. 53 (2006) 270 [INSPIRE].

[75] CMS collaboration, Measurement of the inelastic proton-proton cross section at $\sqrt{s}=13 \mathrm{TeV}$, JHEP 07 (2018) 161 [arXiv: 1802. 02613] [inSPIRE].

[76] D. Krohn, J. Thaler and L.-T. Wang, Jet trimming, JHEP 02 (2010) 084 [arXiv:0912.1342] [INSPIRE].

[77] CMS collaboration, Combination of searches for heavy resonances decaying to $W W, W Z$, $Z Z, W H$ and $Z H$ boson pairs in proton-proton collisions at $\sqrt{s}=8$ and 13 TeV, Phys. Lett. B 774 (2017) 533 [arXiv:1705.09171] [INSPIRE].

[78] J. Thaler and K. Van Tilburg, Maximizing boosted top identification by minimizing $N$-subjettiness, JHEP 02 (2012) 093 [arXiv:1108.2701] [INSPIRE]. 
[79] CMS collaboration, Identification of heavy-flavour jets with the CMS detector in pp collisions at $13 \mathrm{TeV}, 2018$ JINST $13 \mathrm{P} 05011$ [arXiv: 1712.07158] [INSPIRE].

[80] R.G. Lomax and D.L. Hahs-Vaughn, Statistical concepts: a second course, Taylor and Francis, Hoboken, NJ, U.S.A., (2012).

[81] CMS collaboration, CMS luminosity measurements for the 2016 data taking period, CMS-PAS-LUM-17-001, CERN, Geneva, Switzerland, (2017).

[82] R.J. Barlow and C. Beeston, Fitting using finite Monte Carlo samples, Comput. Phys. Commun. 77 (1993) 219 [INSPIRE].

[83] J.S. Conway, Incorporating nuisance parameters in likelihoods for multisource spectra, in Proceedings, PHYSTAT 2011 Workshop on Statistical Issues Related to Discovery Claims in Search Experiments and Unfolding, CERN, Geneva, Switzerland, 17-20 January 2011, pg. 115 [arXiv: 1103.0354] [INSPIRE].

[84] ATLAS, CMS collaborations and LHC Higgs Combination Group, Procedure for the LHC Higgs boson search combination in Summer 2011, CMS-NOTE-2011-005, CERN, Geneva, Switzerland, (2011).

[85] G. Cowan, K. Cranmer, E. Gross and O. Vitells, Asymptotic formulae for likelihood-based tests of new physics, Eur. Phys. J. C 71 (2011) 1554 [Erratum ibid. C 73 (2013) 2501] [arXiv: 1007.1727] [INSPIRE].

[86] A.L. Read, Presentation of search results: the $C L_{s}$ technique, J. Phys. G 28 (2002) 2693 [INSPIRE].

[87] T. Junk, Confidence level computation for combining searches with small statistics, Nucl. Instrum. Meth. A 434 (1999) 435 [hep-ex/9902006] [INSPIRE].

[88] A.L. Fitzpatrick, J. Kaplan, L. Randall and L.-T. Wang, Searching for the Kaluza-Klein graviton in bulk RS models, JHEP 09 (2007) 013 [hep-ph/0701150] [INSPIRE].

[89] A. Oliveira, Gravity particles from warped extra dimensions, predictions for LHC, arXiv: 1404.0102 [INSPIRE]. 


\section{The CMS collaboration}

\section{Yerevan Physics Institute, Yerevan, Armenia}

A.M. Sirunyan, A. Tumasyan

\section{Institut für Hochenergiephysik, Wien, Austria}

W. Adam, F. Ambrogi, E. Asilar, T. Bergauer, J. Brandstetter, M. Dragicevic, J. Erö, A. Escalante Del Valle, M. Flechl, R. Frühwirth ${ }^{1}$, V.M. Ghete, J. Hrubec, M. Jeitler ${ }^{1}$, N. Krammer, I. Krätschmer, D. Liko, T. Madlener, I. Mikulec, N. Rad, H. Rohringer, J. Schieck ${ }^{1}$, R. Schöfbeck, M. Spanring, D. Spitzbart, A. Taurok, W. Waltenberger, J. Wittmann, C.-E. Wulz ${ }^{1}$, M. Zarucki

\section{Institute for Nuclear Problems, Minsk, Belarus}

V. Chekhovsky, V. Mossolov, J. Suarez Gonzalez

Universiteit Antwerpen, Antwerpen, Belgium

E.A. De Wolf, D. Di Croce, X. Janssen, J. Lauwers, M. Pieters, H. Van Haevermaet, P. Van Mechelen, N. Van Remortel

\section{Vrije Universiteit Brussel, Brussel, Belgium}

S. Abu Zeid, F. Blekman, J. D'Hondt, I. De Bruyn, J. De Clercq, K. Deroover, G. Flouris, D. Lontkovskyi, S. Lowette, I. Marchesini, S. Moortgat, L. Moreels, Q. Python, K. Skovpen, S. Tavernier, W. Van Doninck, P. Van Mulders, I. Van Parijs

\section{Université Libre de Bruxelles, Bruxelles, Belgium}

D. Beghin, B. Bilin, H. Brun, B. Clerbaux, G. De Lentdecker, H. Delannoy, B. Dorney, G. Fasanella, L. Favart, R. Goldouzian, A. Grebenyuk, A.K. Kalsi, T. Lenzi, J. Luetic, N. Postiau, E. Starling, L. Thomas, C. Vander Velde, P. Vanlaer, D. Vannerom, Q. Wang

\section{Ghent University, Ghent, Belgium}

T. Cornelis, D. Dobur, A. Fagot, M. Gul, I. Khvastunov², D. Poyraz, C. Roskas, D. Trocino, M. Tytgat, W. Verbeke, B. Vermassen, M. Vit, N. Zaganidis

\section{Université Catholique de Louvain, Louvain-la-Neuve, Belgium}

H. Bakhshiansohi, O. Bondu, S. Brochet, G. Bruno, C. Caputo, P. David, C. Delaere, M. Delcourt, B. Francois, A. Giammanco, G. Krintiras, V. Lemaitre, A. Magitteri, A. Mertens, M. Musich, K. Piotrzkowski, A. Saggio, M. Vidal Marono, S. Wertz, J. Zobec

\section{Centro Brasileiro de Pesquisas Fisicas, Rio de Janeiro, Brazil}

F.L. Alves, G.A. Alves, M. Correa Martins Junior, G. Correia Silva, C. Hensel, A. Moraes, M.E. Pol, P. Rebello Teles

Universidade do Estado do Rio de Janeiro, Rio de Janeiro, Brazil

E. Belchior Batista Das Chagas, W. Carvalho, J. Chinellato ${ }^{3}$, E. Coelho, E.M. Da Costa, G.G. Da Silveira ${ }^{4}$, D. De Jesus Damiao, C. De Oliveira Martins, S. Fonseca De Souza, H. Malbouisson, D. Matos Figueiredo, M. Melo De Almeida, C. Mora Herrera, L. Mundim, H. Nogima, W.L. Prado Da Silva, L.J. Sanchez Rosas, A. Santoro, A. Sznajder, M. Thiel, E.J. Tonelli Manganote ${ }^{3}$, F. Torres Da Silva De Araujo, A. Vilela Pereira 
Universidade Estadual Paulista ${ }^{a}$, Universidade Federal do ABC ${ }^{b}$, São Paulo, Brazil

S. Ahuja ${ }^{a}$, C.A. Bernardes ${ }^{a}$, L. Calligaris $^{a}$, T.R. Fernandez Perez Tomei ${ }^{a}$, E.M. $_{\text {Gregores }}{ }^{b}$, P.G. Mercadante ${ }^{b}$, S.F. Novaes ${ }^{a}$, SandraS. Padula ${ }^{a}$

Institute for Nuclear Research and Nuclear Energy, Bulgarian Academy of Sciences, Sofia, Bulgaria

A. Aleksandrov, R. Hadjiiska, P. Iaydjiev, A. Marinov, M. Misheva, M. Rodozov,

M. Shopova, G. Sultanov

University of Sofia, Sofia, Bulgaria

A. Dimitrov, L. Litov, B. Pavlov, P. Petkov

Beihang University, Beijing, China

W. Fang ${ }^{5}$, X. Gao ${ }^{5}$, L. Yuan

Institute of High Energy Physics, Beijing, China

M. Ahmad, J.G. Bian, G.M. Chen, H.S. Chen, M. Chen, Y. Chen, C.H. Jiang, D. Leggat,

H. Liao, Z. Liu, F. Romeo, S.M. Shaheen ${ }^{6}$, A. Spiezia, J. Tao, Z. Wang, E. Yazgan,

H. Zhang, S. Zhang ${ }^{6}$, J. Zhao

State Key Laboratory of Nuclear Physics and Technology, Peking University, Beijing, China

Y. Ban, G. Chen, A. Levin, J. Li, L. Li, Q. Li, Y. Mao, S.J. Qian, D. Wang, Z. Xu

Tsinghua University, Beijing, China

Y. Wang

Universidad de Los Andes, Bogota, Colombia

C. Avila, A. Cabrera, C.A. Carrillo Montoya, L.F. Chaparro Sierra, C. Florez,

C.F. González Hernández, M.A. Segura Delgado

University of Split, Faculty of Electrical Engineering, Mechanical Engineering and Naval Architecture, Split, Croatia

B. Courbon, N. Godinovic, D. Lelas, I. Puljak, T. Sculac

University of Split, Faculty of Science, Split, Croatia

Z. Antunovic, M. Kovac

Institute Rudjer Boskovic, Zagreb, Croatia

V. Brigljevic, D. Ferencek, K. Kadija, B. Mesic, A. Starodumov ${ }^{7}$, T. Susa

University of Cyprus, Nicosia, Cyprus

M.W. Ather, A. Attikis, M. Kolosova, G. Mavromanolakis, J. Mousa, C. Nicolaou, F. Ptochos, P.A. Razis, H. Rykaczewski

Charles University, Prague, Czech Republic

M. Finger ${ }^{8}$, M. Finger Jr. ${ }^{8}$ 
Escuela Politecnica Nacional, Quito, Ecuador

E. Ayala

Universidad San Francisco de Quito, Quito, Ecuador

E. Carrera Jarrin

Academy of Scientific Research and Technology of the Arab Republic of Egypt, Egyptian Network of High Energy Physics, Cairo, Egypt

H. Abdalla ${ }^{9}$, A. Mahrous ${ }^{10}$, A. Mohamed ${ }^{11}$

National Institute of Chemical Physics and Biophysics, Tallinn, Estonia

S. Bhowmik, A. Carvalho Antunes De Oliveira, R.K. Dewanjee, K. Ehataht, M. Kadastik, M. Raidal, C. Veelken

Department of Physics, University of Helsinki, Helsinki, Finland

P. Eerola, H. Kirschenmann, J. Pekkanen, M. Voutilainen

Helsinki Institute of Physics, Helsinki, Finland

J. Havukainen, J.K. Heikkilä, T. Järvinen, V. Karimäki, R. Kinnunen, T. Lampén, K. Lassila-Perini, S. Laurila, S. Lehti, T. Lindén, P. Luukka, T. Mäenpää, H. Siikonen, E. Tuominen, J. Tuominiemi

\section{Lappeenranta University of Technology, Lappeenranta, Finland}

T. Tuuva

IRFU, CEA, Université Paris-Saclay, Gif-sur-Yvette, France

M. Besancon, F. Couderc, M. Dejardin, D. Denegri, J.L. Faure, F. Ferri, S. Ganjour, A. Givernaud, P. Gras, G. Hamel de Monchenault, P. Jarry, C. Leloup, E. Locci, J. Malcles, G. Negro, J. Rander, A. Rosowsky, M.Ö. Sahin, M. Titov

Laboratoire Leprince-Ringuet, Ecole polytechnique, CNRS/IN2P3, Université Paris-Saclay, Palaiseau, France

A. Abdulsalam ${ }^{12}$, C. Amendola, I. Antropov, F. Beaudette, P. Busson, C. Charlot, R. Granier de Cassagnac, I. Kucher, A. Lobanov, J. Martin Blanco, C. Martin Perez, M. Nguyen, C. Ochando, G. Ortona, P. Paganini, P. Pigard, J. Rembser, R. Salerno, J.B. Sauvan, Y. Sirois, A.G. Stahl Leiton, A. Zabi, A. Zghiche

Université de Strasbourg, CNRS, IPHC UMR 7178, Strasbourg, France J.-L. Agram ${ }^{13}$, J. Andrea, D. Bloch, J.-M. Brom, E.C. Chabert, V. Cherepanov, C. Collard, E. Conte ${ }^{13}$, J.-C. Fontaine ${ }^{13}$, D. Gelé, U. Goerlach, M. Jansová, A.-C. Le Bihan, N. Tonon, P. Van Hove

Centre de Calcul de l'Institut National de Physique Nucleaire et de Physique des Particules, CNRS/IN2P3, Villeurbanne, France

S. Gadrat 
Université de Lyon, Université Claude Bernard Lyon 1, CNRS-IN2P3, Institut de Physique Nucléaire de Lyon, Villeurbanne, France

S. Beauceron, C. Bernet, G. Boudoul, N. Chanon, R. Chierici, D. Contardo, P. Depasse, H. El Mamouni, J. Fay, L. Finco, S. Gascon, M. Gouzevitch, G. Grenier, B. Ille, F. Lagarde, I.B. Laktineh, H. Lattaud, M. Lethuillier, L. Mirabito, S. Perries, A. Popov ${ }^{14}$, V. Sordini, G. Touquet, M. Vander Donckt, S. Viret

Georgian Technical University, Tbilisi, Georgia

A. Khvedelidze ${ }^{8}$

Tbilisi State University, Tbilisi, Georgia

D. Lomidze

RWTH Aachen University, I. Physikalisches Institut, Aachen, Germany

C. Autermann, L. Feld, M.K. Kiesel, K. Klein, M. Lipinski, M. Preuten, M.P. Rauch, C. Schomakers, J. Schulz, M. Teroerde, B. Wittmer, V. Zhukov ${ }^{14}$

RWTH Aachen University, III. Physikalisches Institut A, Aachen, Germany

A. Albert, D. Duchardt, M. Endres, M. Erdmann, S. Ghosh, A. Güth, T. Hebbeker, C. Heidemann, K. Hoepfner, H. Keller, L. Mastrolorenzo, M. Merschmeyer, A. Meyer, P. Millet, S. Mukherjee, T. Pook, M. Radziej, H. Reithler, M. Rieger, A. Schmidt, D. Teyssier

RWTH Aachen University, III. Physikalisches Institut B, Aachen, Germany

G. Flügge, O. Hlushchenko, T. Kress, A. Künsken, T. Müller, A. Nehrkorn, A. Nowack, C. Pistone, O. Pooth, D. Roy, H. Sert, A. Stahl ${ }^{15}$

\section{Deutsches Elektronen-Synchrotron, Hamburg, Germany}

M. Aldaya Martin, T. Arndt, C. Asawatangtrakuldee, I. Babounikau, K. Beernaert, O. Behnke, U. Behrens, A. Bermúdez Martínez, D. Bertsche, A.A. Bin Anuar, K. Borras ${ }^{16}$, V. Botta, A. Campbell, P. Connor, C. Contreras-Campana, V. Danilov, A. De Wit, M.M. Defranchis, C. Diez Pardos, D. Domínguez Damiani, G. Eckerlin, T. Eichhorn, A. Elwood, E. Eren, E. Gallo ${ }^{17}$, A. Geiser, A. Grohsjean, M. Guthoff, M. Haranko, A. Harb, J. Hauk, H. Jung, M. Kasemann, J. Keaveney, C. Kleinwort, J. Knolle, D. Krücker, W. Lange, A. Lelek, T. Lenz, J. Leonard, K. Lipka, W. Lohmann ${ }^{18}$, R. Mankel, I.A. Melzer-Pellmann, A.B. Meyer, M. Meyer, M. Missiroli, G. Mittag, J. Mnich, V. Myronenko, S.K. Pflitsch, D. Pitzl, A. Raspereza, M. Savitskyi, P. Saxena, P. Schütze, C. Schwanenberger, R. Shevchenko, A. Singh, H. Tholen, O. Turkot, A. Vagnerini, G.P. Van Onsem, R. Walsh, Y. Wen, K. Wichmann, C. Wissing, O. Zenaiev

\section{University of Hamburg, Hamburg, Germany}

R. Aggleton, S. Bein, L. Benato, A. Benecke, V. Blobel, T. Dreyer, E. Garutti, D. Gonzalez, P. Gunnellini, J. Haller, A. Hinzmann, A. Karavdina, G. Kasieczka, R. Klanner, R. Kogler, N. Kovalchuk, S. Kurz, V. Kutzner, J. Lange, D. Marconi, J. Multhaup, M. Niedziela, C.E.N. Niemeyer, D. Nowatschin, A. Perieanu, A. Reimers, O. Rieger, C. Scharf, P. Schleper, S. Schumann, J. Schwandt, J. Sonneveld, H. Stadie, G. Steinbrück, F.M. Stober, M. Stöver, A. Vanhoefer, B. Vormwald, I. Zoi 
Karlsruher Institut fuer Technology

M. Akbiyik, C. Barth, M. Baselga, S. Baur, E. Butz, R. Caspart, T. Chwalek, F. Colombo, W. De Boer, A. Dierlamm, K. El Morabit, N. Faltermann, B. Freund, M. Giffels, M.A. Harrendorf, F. Hartmann ${ }^{15}$, S.M. Heindl, U. Husemann, F. Kassel ${ }^{15}$, I. Katkov ${ }^{14}$, S. Kudella, H. Mildner, S. Mitra, M.U. Mozer, Th. Müller, M. Plagge, G. Quast, K. Rabbertz, M. Schröder, I. Shvetsov, G. Sieber, H.J. Simonis, R. Ulrich, S. Wayand, M. Weber, T. Weiler, S. Williamson, C. Wöhrmann, R. Wolf

Institute of Nuclear and Particle Physics (INPP), NCSR Demokritos, Aghia Paraskevi, Greece

G. Anagnostou, G. Daskalakis, T. Geralis, A. Kyriakis, D. Loukas, G. Paspalaki, I. TopsisGiotis

National and Kapodistrian University of Athens, Athens, Greece

G. Karathanasis, S. Kesisoglou, P. Kontaxakis, A. Panagiotou, I. Papavergou, N. Saoulidou, E. Tziaferi, K. Vellidis

National Technical University of Athens, Athens, Greece

K. Kousouris, I. Papakrivopoulos, G. Tsipolitis

University of Ioánnina, Ioánnina, Greece

I. Evangelou, C. Foudas, P. Gianneios, P. Katsoulis, P. Kokkas, S. Mallios, N. Manthos, I. Papadopoulos, E. Paradas, J. Strologas, F.A. Triantis, D. Tsitsonis

MTA-ELTE Lendület CMS Particle and Nuclear Physics Group, Eötvös Loránd University, Budapest, Hungary

M. Bartók ${ }^{19}$, M. Csanad, N. Filipovic, P. Major, M.I. Nagy, G. Pasztor, O. Surányi, G.I. Veres

Wigner Research Centre for Physics, Budapest, Hungary

G. Bencze, C. Hajdu, D. Horvath ${ }^{20}$, Á. Hunyadi, F. Sikler, T.Á. Vámi, V. Veszpremi, G. Vesztergombi ${ }^{\dagger}$

Institute of Nuclear Research ATOMKI, Debrecen, Hungary

N. Beni, S. Czellar, J. Karancsi21 ${ }^{21}$ A. Makovec, J. Molnar, Z. Szillasi

Institute of Physics, University of Debrecen, Debrecen, Hungary

P. Raics, Z.L. Trocsanyi, B. Ujvari

Indian Institute of Science (IISc), Bangalore, India

S. Choudhury, J.R. Komaragiri, P.C. Tiwari

National Institute of Science Education and Research, HBNI, Bhubaneswar, India

S. Bahinipati22 , C. Kar, P. Mal, K. Mandal, A. $\operatorname{Nayak}^{23}$, D.K. Sahoo ${ }^{22}$, S.K. Swain 
Panjab University, Chandigarh, India

S. Bansal, S.B. Beri, V. Bhatnagar, S. Chauhan, R. Chawla, N. Dhingra, R. Gupta, A. Kaur, M. Kaur, S. Kaur, R. Kumar, P. Kumari, M. Lohan, A. Mehta, K. Sandeep, S. Sharma, J.B. Singh, A.K. Virdi, G. Walia

\section{University of Delhi, Delhi, India}

A. Bhardwaj, B.C. Choudhary, R.B. Garg, M. Gola, S. Keshri, Ashok Kumar, S. Malhotra, M. Naimuddin, P. Priyanka, K. Ranjan, Aashaq Shah, R. Sharma

Saha Institute of Nuclear Physics, HBNI, Kolkata, India

R. Bhardwaj 24, M. Bharti24, R. Bhattacharya, S. Bhattacharya, U. Bhawandeep ${ }^{24}$, D. Bhowmik, S. Dey, S. Dutt ${ }^{24}$, S. Dutta, S. Ghosh, K. Mondal, S. Nandan, A. Purohit, P.K. Rout, A. Roy, S. Roy Chowdhury, G. Saha, S. Sarkar, M. Sharan, B. Singh ${ }^{24}$, S. Thakur ${ }^{24}$

\section{Indian Institute of Technology Madras, Madras, India}

P.K. Behera

\section{Bhabha Atomic Research Centre, Mumbai, India}

R. Chudasama, D. Dutta, V. Jha, V. Kumar, P.K. Netrakanti, L.M. Pant, P. Shukla

Tata Institute of Fundamental Research-A, Mumbai, India

T. Aziz, M.A. Bhat, S. Dugad, G.B. Mohanty, N. Sur, B. Sutar, RavindraKumar Verma

Tata Institute of Fundamental Research-B, Mumbai, India

S. Banerjee, S. Bhattacharya, S. Chatterjee, P. Das, M. Guchait, Sa. Jain, S. Karmakar, S. Kumar, M. Maity ${ }^{25}$, G. Majumder, K. Mazumdar, N. Sahoo, T. Sarkar ${ }^{25}$

Indian Institute of Science Education and Research (IISER), Pune, India

S. Chauhan, S. Dube, V. Hegde, A. Kapoor, K. Kothekar, S. Pandey, A. Rane, S. Sharma

\section{Institute for Research in Fundamental Sciences (IPM), Tehran, Iran}

S. Chenarani ${ }^{26}$, E. Eskandari Tadavani, S.M. Etesami ${ }^{26}$, M. Khakzad, M. Mohammadi Najafabadi, M. Naseri, F. Rezaei Hosseinabadi, B. Safarzadeh ${ }^{27}$, M. Zeinali

\section{University College Dublin, Dublin, Ireland}

M. Felcini, M. Grunewald

INFN Sezione di Bari ${ }^{a}$, Università di Bari ${ }^{b}$, Politecnico di Bari ${ }^{c}$, Bari, Italy M. Abbrescia ${ }^{a, b}$, C. Calabria ${ }^{a, b}$, A. Colaleo ${ }^{a}$, D. Creanza ${ }^{a, c}$, L. Cristella ${ }^{a, b}$, N. De Filippis ${ }^{a, c}$, M. De Palma ${ }^{a, b}$, A. Di Florio ${ }^{a, b}$, F. Errico ${ }^{a, b}$, L. Fiore $^{a}$, A. Gelmi ${ }^{a, b}$, G. Iaselli ${ }^{a, c}$, M. Ince ${ }^{a, b}$, S. Lezki ${ }^{a, b}$, G. Maggi ${ }^{a, c}$, M. Maggi ${ }^{a}$, G. Miniello ${ }^{a, b}, \mathrm{~S}_{\text {. My }}{ }^{a, b}$, S. Nuzzo ${ }^{a, b}$, A. Pompilia, ${ }^{a, b}$ G. Pugliese ${ }^{a, c}$, R. Radogna ${ }^{a}$, A. Ranieri ${ }^{a}$, G. Selvaggi ${ }^{a}, b$, A. Sharma ${ }^{a}$, L. Silvestris ${ }^{a}$, R. Venditti ${ }^{a}$, P. Verwilligen ${ }^{a}$, G. Zito ${ }^{a}$

INFN Sezione di Bologna ${ }^{a}$, Università di Bologna ${ }^{b}$, Bologna, Italy

G. Abbiendi ${ }^{a}$, C. Battilana ${ }^{a, b}$, D. Bonacorsi ${ }^{a}, b$, L. Borgonovi $^{a, b}$, S. Braibant-Giacomelli ${ }^{a, b}$,

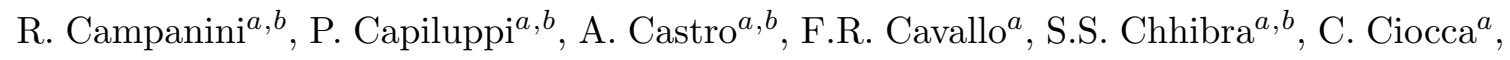
G. Codispoti ${ }^{a, b}$, M. Cuffiani ${ }^{a, b}$, G.M. Dallavalle ${ }^{a}$, F. Fabbri ${ }^{a}$, A. Fanfani ${ }^{a, b}$, E. Fontanesi, 
P. Giacomelli ${ }^{a}$, C. Grandi ${ }^{a}$, L. Guiducci ${ }^{a}, b$, S. Lo Meo ${ }^{a}$, S. Marcellini ${ }^{a}$, G. Masetti ${ }^{a}$, A. Montanari ${ }^{a}$, F.L. Navarria ${ }^{a, b}$, A. Perrotta ${ }^{a}$, F. Primavera ${ }^{a, b, 15}$, A.M. Rossi ${ }^{a, b}$, T. Rovelli ${ }^{a, b}$, G.P. Siroli ${ }^{a, b}$, N. Tosi ${ }^{a}$

INFN Sezione di Catania ${ }^{a}$, Università di Catania ${ }^{b}$, Catania, Italy

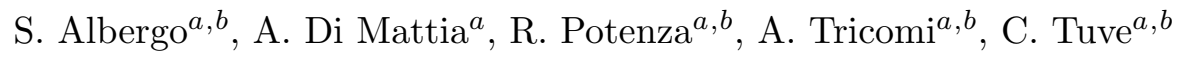

INFN Sezione di Firenze ${ }^{a}$, Università di Firenze ${ }^{b}$, Firenze, Italy

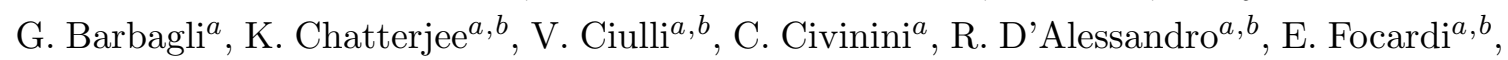

G. Latino, P. Lenzi ${ }^{a, b}$, M. Meschini ${ }^{a}$, S. Paoletti ${ }^{a}$, L. Russo $^{a}, 28$, G. Sguazzoni $^{a}$, D. Strom ${ }^{a}$,

L. Viliani ${ }^{a}$

INFN Laboratori Nazionali di Frascati, Frascati, Italy

L. Benussi, S. Bianco, F. Fabbri, D. Piccolo

INFN Sezione di Genova ${ }^{a}$, Università di Genova ${ }^{b}$, Genova, Italy

F. Ferro $^{a}$, F. Ravera ${ }^{a, b}$, E. Robutti $^{a}$, S. Tosi ${ }^{a, b}$

INFN Sezione di Milano-Bicocca ${ }^{a}$, Università di Milano-Bicocca ${ }^{b}$, Milano, Italy

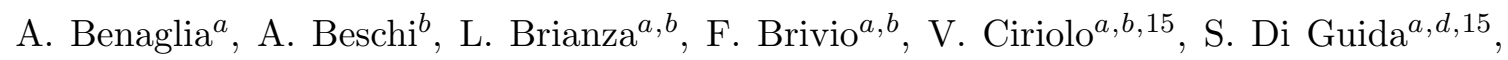
M.E. Dinardo ${ }^{a, b}$, S. Fiorendi ${ }^{a}, b$ S. Gennai ${ }^{a}$, A. Ghezzi ${ }^{a, b}$, P. Govoni $^{a, b}$, M. Malberti ${ }^{a, b}$, S. Malvezzi ${ }^{a}$, A. Massironi ${ }^{a, b}$, D. Menasce ${ }^{a}$, F. Monti, L. Moroni ${ }^{a}$, M. Paganoni ${ }^{a, b}$, D. Pedrini ${ }^{a}$, S. Ragazzi ${ }^{a, b}$, T. Tabarelli de Fatis ${ }^{a, b}$, D. Zuolo ${ }^{a, b}$

INFN Sezione di Napoli ${ }^{a}$, Università di Napoli 'Federico II' ${ }^{b}$, Napoli, Italy, Università della Basilicata ${ }^{c}$, Potenza, Italy, Università G. Marconi ${ }^{d}$, Roma, Italy

S. Buontempo ${ }^{a}$, N. Cavallo ${ }^{a, c}$, A. Di Crescenzo ${ }^{a, b}$, F. Fabozzi $^{a, c}$, F. Fienga $^{a}$, G. Galati $^{a}$,

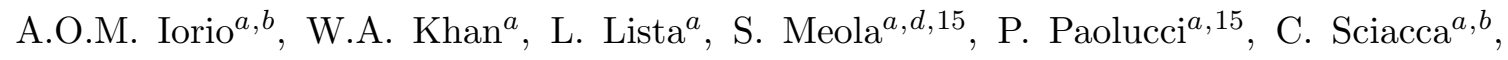
E. Voevodina ${ }^{a, b}$

INFN Sezione di Padova ${ }^{a}$, Università di Padova ${ }^{b}$, Padova, Italy, Università di Trento ${ }^{c}$, Trento, Italy

P. Azzi ${ }^{a}$, N. Bacchetta ${ }^{a}$, D. Bisello ${ }^{a, b}$, A. Boletti ${ }^{a, b}$, A. Bragagnolo, R. Carlin ${ }^{a, b}$, P. Checchia ${ }^{a}$, M. Dall'Osso ${ }^{a, b}$, P. De Castro Manzano ${ }^{a}$, T. Dorigo ${ }^{a}$, U. Dosselli ${ }^{a}$, F. Gasparini ${ }^{a, b}$, U. Gasparini ${ }^{a, b}$, A. Gozzelino ${ }^{a}$, S.Y. Hoh, S. Lacaprara ${ }^{a}$, P. Lujan, M. Margoni ${ }^{a, b}$, A.T. Meneguzzo ${ }^{a, b}$, J. Pazzini ${ }^{a, b}$, P. Ronchese ${ }^{a, b}$, R. $\operatorname{Rossin}^{a, b}$, F. Simonetto ${ }^{a, b}$, A. Tiko, E. Torassa ${ }^{a}$, M. Zanetti ${ }^{a, b}$, P. Zotto ${ }^{a, b}$, G. Zumerle ${ }^{a, b}$

INFN Sezione di Pavia ${ }^{a}$, Università di Pavia ${ }^{b}$, Pavia, Italy

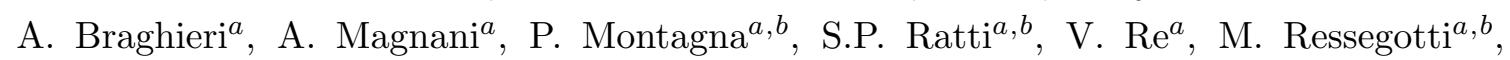
C. Riccardi ${ }^{a}, b$ P. Salvini ${ }^{a}$, I. Vai $^{a, b}$, P. Vitulo ${ }^{a, b}$

INFN Sezione di Perugia ${ }^{a}$, Università di Perugia ${ }^{b}$, Perugia, Italy

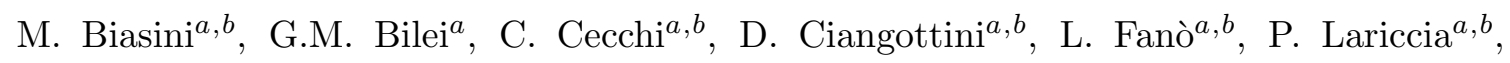

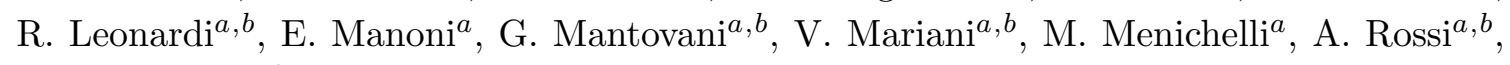
A. Santocchia ${ }^{a, b}$, D. Spiga ${ }^{a}$ 
INFN Sezione di Pisa ${ }^{a}$, Università di Pisa ${ }^{b}$, Scuola Normale Superiore di Pisa ${ }^{c}$, Pisa, Italy

K. Androsov ${ }^{a}$, P. Azzurri ${ }^{a}$, G. Bagliesi ${ }^{a}$, L. Bianchini ${ }^{a}$, T. Boccali ${ }^{a}$, L. Borrello, R. Castaldi ${ }^{a}$, M.A. Ciocci ${ }^{a}, b$, R. Dell'Orso ${ }^{a}$, G. Fedi ${ }^{a}$, F. Fiori ${ }^{a, c}$, L. Giannini $^{a, c}$, A. Giassi $^{a}$,

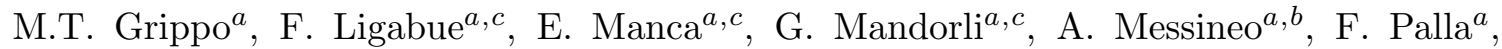

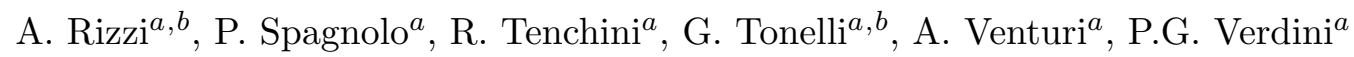

INFN Sezione di Roma ${ }^{a}$, Sapienza Università di Roma ${ }^{b}$, Rome, Italy

L. Barone ${ }^{a, b}$, F. Cavallari ${ }^{a}$, M. Cipriani ${ }^{a, b}$, D. Del Re ${ }^{a, b}$, E. Di Marco ${ }^{a, b}$, M. Diemoz $^{a}$, S. Gelli ${ }^{a, b}$, E. Longo ${ }^{a, b}$, B. Marzocchi ${ }^{a}, b$, P. Meridiani ${ }^{a}$, G. Organtini ${ }^{a, b}$, F. Pandolfi ${ }^{a}$,

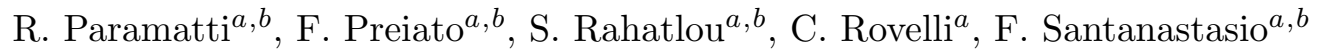

INFN Sezione di Torino ${ }^{a}$, Università di Torino ${ }^{b}$, Torino, Italy, Università del Piemonte Orientale ${ }^{c}$, Novara, Italy

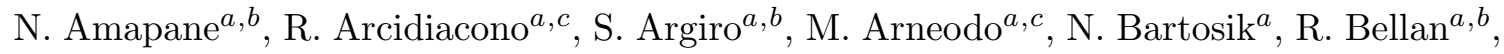

C. Biino ${ }^{a}$, N. Cartiglia ${ }^{a}$, F. Cenna ${ }^{a, b}$, S. Cometti ${ }^{a}$, M. Costa ${ }^{a, b}$, R. Covarelli ${ }^{a, b}$,

N. Demaria ${ }^{a}$, B. Kiani ${ }^{a, b}$, C. Mariotti ${ }^{a}$, S. Maselli ${ }^{a}$, E. Migliore ${ }^{a, b}$, V. Monaco ${ }^{a, b}$,

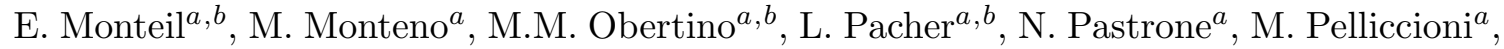
G.L. Pinna Angioni ${ }^{a, b}$, A. Romero ${ }^{a, b}$, M. Ruspa ${ }^{a, c}$, R. Sacchi ${ }^{a, b}$, K. Shchelina ${ }^{a, b}$, V. Sola ${ }^{a}$, A. Solano ${ }^{a, b}$, D. Soldi ${ }^{a, b}$, A. Staiano ${ }^{a}$

INFN Sezione di Trieste ${ }^{a}$, Università di Trieste ${ }^{b}$, Trieste, Italy

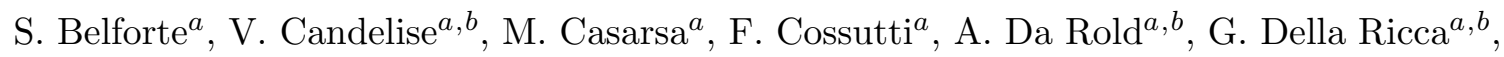
F. Vazzoler ${ }^{a, b}$, A. Zanetti ${ }^{a}$

Kyungpook National University

D.H. Kim, G.N. Kim, M.S. Kim, J. Lee, S. Lee, S.W. Lee, C.S. Moon, Y.D. Oh, S.I. Pak, S. Sekmen, D.C. Son, Y.C. Yang

Chonnam National University, Institute for Universe and Elementary Particles, Kwangju, Korea

H. Kim, D.H. Moon, G. Oh

Hanyang University, Seoul, Korea

J. Goh ${ }^{29}$, T.J. Kim

Korea University, Seoul, Korea

S. Cho, S. Choi, Y. Go, D. Gyun, S. Ha, B. Hong, Y. Jo, K. Lee, K.S. Lee, S. Lee, J. Lim, S.K. Park, Y. Roh

Sejong University, Seoul, Korea

H.S. Kim

Seoul National University, Seoul, Korea

J. Almond, J. Kim, J.S. Kim, H. Lee, K. Lee, K. Nam, S.B. Oh, B.C. Radburn-Smith, S.h. Seo, U.K. Yang, H.D. Yoo, G.B. Yu

University of Seoul, Seoul, Korea

D. Jeon, H. Kim, J.H. Kim, J.S.H. Lee, I.C. Park 
Sungkyunkwan University, Suwon, Korea

Y. Choi, C. Hwang, J. Lee, I. Yu

Vilnius University, Vilnius, Lithuania

V. Dudenas, A. Juodagalvis, J. Vaitkus

National Centre for Particle Physics, Universiti Malaya, Kuala Lumpur, Malaysia

I. Ahmed, Z.A. Ibrahim, M.A.B. Md $\mathrm{Ali}^{30}$, F. Mohamad Idris ${ }^{31}$, W.A.T. Wan Abdullah, M.N. Yusli, Z. Zolkapli

Universidad de Sonora (UNISON), Hermosillo, Mexico

J.F. Benitez, A. Castaneda Hernandez, J.A. Murillo Quijada

Centro de Investigacion y de Estudios Avanzados del IPN, Mexico City, Mexico

H. Castilla-Valdez, E. De La Cruz-Burelo, M.C. Duran-Osuna, I. Heredia-De La Cruz ${ }^{32}$,

R. Lopez-Fernandez, J. Mejia Guisao, R.I. Rabadan-Trejo, M. Ramirez-Garcia,

G. Ramirez-Sanchez, R Reyes-Almanza, A. Sanchez-Hernandez

Universidad Iberoamericana, Mexico City, Mexico

S. Carrillo Moreno, C. Oropeza Barrera, F. Vazquez Valencia

Benemerita Universidad Autonoma de Puebla, Puebla, Mexico

J. Eysermans, I. Pedraza, H.A. Salazar Ibarguen, C. Uribe Estrada

Universidad Autónoma de San Luis Potosí, San Luis Potosí, Mexico

A. Morelos Pineda

University of Auckland, Auckland, New Zealand

D. Krofcheck

University of Canterbury, Christchurch, New Zealand

S. Bheesette, P.H. Butler

National Centre for Physics, Quaid-I-Azam University, Islamabad, Pakistan

A. Ahmad, M. Ahmad, M.I. Asghar, Q. Hassan, H.R. Hoorani, A. Saddique, M.A. Shah, M. Shoaib, M. Waqas

National Centre for Nuclear Research, Swierk, Poland

H. Bialkowska, M. Bluj, B. Boimska, T. Frueboes, M. Górski, M. Kazana, K. Nawrocki, M. Szleper, P. Traczyk, P. Zalewski

Institute of Experimental Physics, Faculty of Physics, University of Warsaw, Warsaw, Poland

K. Bunkowski, A. Byszuk ${ }^{33}$, K. Doroba, A. Kalinowski, M. Konecki, J. Krolikowski, M. Misiura, M. Olszewski, A. Pyskir, M. Walczak 
Laboratório de Instrumentação e Física Experimental de Partículas, Lisboa, Portugal

M. Araujo, P. Bargassa, C. Beirão Da Cruz E Silva, A. Di Francesco, P. Faccioli, B. Galinhas, M. Gallinaro, J. Hollar, N. Leonardo, M.V. Nemallapudi, J. Seixas, G. Strong, O. Toldaiev, D. Vadruccio, J. Varela

\section{Joint Institute for Nuclear Research, Dubna, Russia}

S. Afanasiev, P. Bunin, M. Gavrilenko, I. Golutvin, I. Gorbunov, A. Kamenev, V. Karjavine, A. Lanev, A. Malakhov, V. Matveev ${ }^{34,35}$, P. Moisenz, V. Palichik, V. Perelygin, S. Shmatov, S. Shulha, N. Skatchkov, V. Smirnov, N. Voytishin, A. Zarubin

Petersburg Nuclear Physics Institute, Gatchina (St. Petersburg), Russia

V. Golovtsov, Y. Ivanov, V. Kim ${ }^{36}$, E. Kuznetsova ${ }^{37}$, P. Levchenko, V. Murzin, V. Oreshkin, I. Smirnov, D. Sosnov, V. Sulimov, L. Uvarov, S. Vavilov, A. Vorobyev

Institute for Nuclear Research, Moscow, Russia

Yu. Andreev, A. Dermenev, S. Gninenko, N. Golubev, A. Karneyeu, M. Kirsanov, N. Krasnikov, A. Pashenkov, D. Tlisov, A. Toropin

Institute for Theoretical and Experimental Physics, Moscow, Russia

V. Epshteyn, V. Gavrilov, N. Lychkovskaya, V. Popov, I. Pozdnyakov, G. Safronov, A. Spiridonov, A. Stepennov, V. Stolin, M. Toms, E. Vlasov, A. Zhokin

Moscow Institute of Physics and Technology, Moscow, Russia

T. Aushev

National Research Nuclear University 'Moscow Engineering Physics Institute' (MEPhI), Moscow, Russia

R. Chistov ${ }^{38}$, M. Danilov ${ }^{38}$, P. Parygin, D. Philippov, S. Polikarpov ${ }^{38}$, E. Tarkovskii

P.N. Lebedev Physical Institute, Moscow, Russia

V. Andreev, M. Azarkin ${ }^{35}$, I. Dremin ${ }^{35}$, M. Kirakosyan ${ }^{35}$, S.V. Rusakov, A. Terkulov

Skobeltsyn Institute of Nuclear Physics, Lomonosov Moscow State University, Moscow, Russia

A. Baskakov, A. Belyaev, E. Boos, V. Bunichev, M. Dubinin ${ }^{39}$, L. Dudko, A. Ershov, A. Gribushin, V. Klyukhin, O. Kodolova, I. Lokhtin, I. Miagkov, S. Obraztsov, S. Petrushanko, V. Savrin

Novosibirsk State University (NSU), Novosibirsk, Russia

A. Barnyakov ${ }^{40}$, V. Blinov ${ }^{40}$, T. Dimova ${ }^{40}$, L. Kardapoltsev ${ }^{40}$, Y. Skovpen ${ }^{40}$

State Research Center of Russian Federation, Institute for High Energy Physics of NRC "Kurchatov Institute", Protvino, Russia

I. Azhgirey, I. Bayshev, S. Bitioukov, D. Elumakhov, A. Godizov, V. Kachanov, A. Kalinin,

D. Konstantinov, P. Mandrik, V. Petrov, R. Ryutin, S. Slabospitskii, A. Sobol, S. Troshin, N. Tyurin, A. Uzunian, A. Volkov 
National Research Tomsk Polytechnic University, Tomsk, Russia

A. Babaev, S. Baidali, V. Okhotnikov

University of Belgrade, Faculty of Physics and Vinca Institute of Nuclear Sciences, Belgrade, Serbia

P. Adzic ${ }^{41}$, P. Cirkovic, D. Devetak, M. Dordevic, J. Milosevic

Centro de Investigaciones Energéticas Medioambientales y Tecnológicas (CIEMAT), Madrid, Spain

J. Alcaraz Maestre, A. Álvarez Fernández, I. Bachiller, M. Barrio Luna, J.A. Brochero Cifuentes, M. Cerrada, N. Colino, B. De La Cruz, A. Delgado Peris, C. Fernandez Bedoya, J.P. Fernández Ramos, J. Flix, M.C. Fouz, O. Gonzalez Lopez, S. Goy Lopez, J.M. Hernandez, M.I. Josa, D. Moran, A. Pérez-Calero Yzquierdo, J. Puerta Pelayo, I. Redondo, L. Romero, M.S. Soares, A. Triossi

Universidad Autónoma de Madrid, Madrid, Spain

C. Albajar, J.F. de Trocóniz

Universidad de Oviedo, Oviedo, Spain

J. Cuevas, C. Erice, J. Fernandez Menendez, S. Folgueras, I. Gonzalez Caballero, J.R. González Fernández, E. Palencia Cortezon, V. Rodríguez Bouza, S. Sanchez Cruz, P. Vischia, J.M. Vizan Garcia

Instituto de Física de Cantabria (IFCA), CSIC-Universidad de Cantabria, Santander, Spain

I.J. Cabrillo, A. Calderon, B. Chazin Quero, J. Duarte Campderros, M. Fernandez, P.J. Fernández Manteca, A. García Alonso, J. Garcia-Ferrero, G. Gomez, A. Lopez Virto, J. Marco, C. Martinez Rivero, P. Martinez Ruiz del Arbol, F. Matorras, J. Piedra Gomez, C. Prieels, T. Rodrigo, A. Ruiz-Jimeno, L. Scodellaro, N. Trevisani, I. Vila, R. Vilar Cortabitarte

University of Ruhuna, Department of Physics, Matara, Sri Lanka

N. Wickramage

CERN, European Organization for Nuclear Research, Geneva, Switzerland

D. Abbaneo, B. Akgun, E. Auffray, G. Auzinger, P. Baillon, A.H. Ball, D. Barney, J. Bendavid, M. Bianco, A. Bocci, C. Botta, E. Brondolin, T. Camporesi, M. Cepeda, G. Cerminara, E. Chapon, Y. Chen, G. Cucciati, D. d'Enterria, A. Dabrowski, N. Daci, V. Daponte, A. David, A. De Roeck, N. Deelen, M. Dobson, M. Dünser, N. Dupont, A. Elliott-Peisert, P. Everaerts, F. Fallavollita ${ }^{42}$, D. Fasanella, G. Franzoni, J. Fulcher, W. Funk, D. Gigi, A. Gilbert, K. Gill, F. Glege, M. Guilbaud, D. Gulhan, J. Hegeman, C. Heidegger, V. Innocente, A. Jafari, P. Janot, O. Karacheban ${ }^{18}$, J. Kieseler, A. Kornmayer, M. Krammer ${ }^{1}$, C. Lange, P. Lecoq, C. Lourenço, L. Malgeri, M. Mannelli, F. Meijers, J.A. Merlin, S. Mersi, E. Meschi, P. Milenovic ${ }^{43}$, F. Moortgat, M. Mulders, J. Ngadiuba, S. Nourbakhsh, S. Orfanelli, L. Orsini, F. Pantaleo ${ }^{15}$, L. Pape, E. Perez, M. Peruzzi, A. Petrilli, G. Petrucciani, A. Pfeiffer, M. Pierini, F.M. Pitters, D. Rabady, A. Racz, T. Reis, G. Rolandi ${ }^{44}$, M. Rovere, H. Sakulin, C. Schäfer, C. Schwick, M. Seidel, 
M. Selvaggi, A. Sharma, P. Silva, P. Sphicas ${ }^{45}$, A. Stakia, J. Steggemann, M. Tosi, D. Treille, A. Tsirou, V. Veckalns ${ }^{46}$, M. Verzetti, W.D. Zeuner

Paul Scherrer Institut, Villigen, Switzerland

L. Caminada ${ }^{47}$, K. Deiters, W. Erdmann, R. Horisberger, Q. Ingram, H.C. Kaestli, D. Kotlinski, U. Langenegger, T. Rohe, S.A. Wiederkehr

ETH Zurich - Institute for Particle Physics and Astrophysics (IPA), Zurich, Switzerland

M. Backhaus, L. Bäni, P. Berger, N. Chernyavskaya, G. Dissertori, M. Dittmar, M. Donegà, C. Dorfer, T.A. Gómez Espinosa, C. Grab, D. Hits, T. Klijnsma, W. Lustermann, R.A. Manzoni, M. Marionneau, M.T. Meinhard, F. Micheli, P. Musella, F. NessiTedaldi, J. Pata, F. Pauss, G. Perrin, L. Perrozzi, S. Pigazzini, M. Quittnat, D. Ruini, D.A. Sanz Becerra, M. Schönenberger, L. Shchutska, V.R. Tavolaro, K. Theofilatos, M.L. Vesterbacka Olsson, R. Wallny, D.H. Zhu

Universität Zürich, Zurich, Switzerland

T.K. Aarrestad, C. Amsler ${ }^{48}$, D. Brzhechko, M.F. Canelli, A. De Cosa, R. Del Burgo, S. Donato, C. Galloni, T. Hreus, B. Kilminster, S. Leontsinis, I. Neutelings, D. Pinna, G. Rauco, P. Robmann, D. Salerno, K. Schweiger, C. Seitz, Y. Takahashi, A. Zucchetta

National Central University, Chung-Li, Taiwan

Y.H. Chang, K.y. Cheng, T.H. Doan, Sh. Jain, R. Khurana, C.M. Kuo, W. Lin, A. Pozdnyakov, S.S. Yu

National Taiwan University (NTU), Taipei, Taiwan

P. Chang, Y. Chao, K.F. Chen, P.H. Chen, W.-S. Hou, Arun Kumar, Y.F. Liu, R.-S. Lu, E. Paganis, A. Psallidas, A. Steen

Chulalongkorn University, Faculty of Science, Department of Physics, Bangkok, Thailand

B. Asavapibhop, N. Srimanobhas, N. Suwonjandee

Çukurova University, Physics Department, Science and Art Faculty, Adana, Turkey

A. Bat, F. Boran, S. Cerci ${ }^{49}$, S. Damarseckin, Z.S. Demiroglu, F. Dolek, C. Dozen, I. Dumanoglu, S. Girgis, G. Gokbulut, Y. Guler, E. Gurpinar, I. Hos ${ }^{50}$, C. Isik, E.E. Kangal ${ }^{51}$, O. Kara, A. Kayis Topaksu, U. Kiminsu, M. Oglakci, G. Onengut, K. Ozdemir ${ }^{52}$, S. Ozturk ${ }^{53}$, D. Sunar Cerci ${ }^{49}$, B. Tali ${ }^{49}$, U.G. Tok, S. Turkcapar, I.S. Zorbakir, C. Zorbilmez

Middle East Technical University, Physics Department, Ankara, Turkey

B. Isildak ${ }^{54}$, G. Karapinar ${ }^{55}$, M. Yalvac, M. Zeyrek

Bogazici University, Istanbul, Turkey

I.O. Atakisi, E. Gülmez, M. Kaya ${ }^{56}$, O. Kaya ${ }^{57}$, S. Ozkorucuklu ${ }^{58}$, S. Tekten, E.A. Yetkin ${ }^{59}$

Istanbul Technical University, Istanbul, Turkey

M.N. Agaras, A. Cakir, K. Cankocak, Y. Komurcu, S. Sen ${ }^{60}$ 
Institute for Scintillation Materials of National Academy of Science of Ukraine, Kharkov, Ukraine

B. Grynyov

National Scientific Center, Kharkov Institute of Physics and Technology, Kharkov, Ukraine

L. Levchuk

University of Bristol, Bristol, United Kingdom

F. Ball, L. Beck, J.J. Brooke, D. Burns, E. Clement, D. Cussans, O. Davignon, H. Flacher,

J. Goldstein, G.P. Heath, H.F. Heath, L. Kreczko, D.M. Newbold ${ }^{61}$, S. Paramesvaran,

B. Penning, T. Sakuma, D. Smith, V.J. Smith, J. Taylor, A. Titterton

Rutherford Appleton Laboratory, Didcot, United Kingdom

K.W. Bell, A. Belyaev ${ }^{62}$, C. Brew, R.M. Brown, D. Cieri, D.J.A. Cockerill, J.A. Coughlan,

K. Harder, S. Harper, J. Linacre, E. Olaiya, D. Petyt, C.H. Shepherd-Themistocleous,

A. Thea, I.R. Tomalin, T. Williams, W.J. Womersley

\section{Imperial College, London, United Kingdom}

R. Bainbridge, P. Bloch, J. Borg, S. Breeze, O. Buchmuller, A. Bundock, D. Colling,

P. Dauncey, G. Davies, M. Della Negra, R. Di Maria, Y. Haddad, G. Hall, G. Iles, T. James, M. Komm, C. Laner, L. Lyons, A.-M. Magnan, S. Malik, A. Martelli, J. Nash ${ }^{63}$, A. Nikitenko ${ }^{7}$, V. Palladino, M. Pesaresi, A. Richards, A. Rose, E. Scott, C. Seez, A. Shtipliyski, G. Singh, M. Stoye, T. Strebler, S. Summers, A. Tapper, K. Uchida, T. Virdee ${ }^{15}$, N. Wardle, D. Winterbottom, J. Wright, S.C. Zenz

Brunel University, Uxbridge, United Kingdom

J.E. Cole, P.R. Hobson, A. Khan, P. Kyberd, C.K. Mackay, A. Morton, I.D. Reid, L. Teodorescu, S. Zahid

Baylor University, Waco, U.S.A.

K. Call, J. Dittmann, K. Hatakeyama, H. Liu, C. Madrid, B. Mcmaster, N. Pastika, C. Smith

Catholic University of America, Washington DC, U.S.A.

R. Bartek, A. Dominguez

The University of Alabama, Tuscaloosa, U.S.A.

A. Buccilli, S.I. Cooper, C. Henderson, P. Rumerio, C. West

Boston University, Boston, U.S.A.

D. Arcaro, T. Bose, D. Gastler, D. Rankin, C. Richardson, J. Rohlf, L. Sulak, D. Zou

Brown University, Providence, U.S.A.

G. Benelli, X. Coubez, D. Cutts, M. Hadley, J. Hakala, U. Heintz, J.M. Hogan ${ }^{64}$, K.H.M. Kwok, E. Laird, G. Landsberg, J. Lee, Z. Mao, M. Narain, S. Sagir ${ }^{65}$, R. Syarif, E. Usai, D. Yu 
University of California, Davis, Davis, U.S.A.

R. Band, C. Brainerd, R. Breedon, D. Burns, M. Calderon De La Barca Sanchez, M. Chertok, J. Conway, R. Conway, P.T. Cox, R. Erbacher, C. Flores, G. Funk, W. Ko, O. Kukral, R. Lander, M. Mulhearn, D. Pellett, J. Pilot, S. Shalhout, M. Shi, D. Stolp, D. Taylor, K. Tos, M. Tripathi, Z. Wang, F. Zhang

University of California, Los Angeles, U.S.A.

M. Bachtis, C. Bravo, R. Cousins, A. Dasgupta, A. Florent, J. Hauser, M. Ignatenko, N. Mccoll, S. Regnard, D. Saltzberg, C. Schnaible, V. Valuev

University of California, Riverside, Riverside, U.S.A.

E. Bouvier, K. Burt, R. Clare, J.W. Gary, S.M.A. Ghiasi Shirazi, G. Hanson, G. Karapostoli, E. Kennedy, F. Lacroix, O.R. Long, M. Olmedo Negrete, M.I. Paneva, W. Si, L. Wang, H. Wei, S. Wimpenny, B.R. Yates

University of California, San Diego, La Jolla, U.S.A.

J.G. Branson, P. Chang, S. Cittolin, M. Derdzinski, R. Gerosa, D. Gilbert, B. Hashemi, A. Holzner, D. Klein, G. Kole, V. Krutelyov, J. Letts, M. Masciovecchio, D. Olivito, S. Padhi, M. Pieri, M. Sani, V. Sharma, S. Simon, M. Tadel, A. Vartak, S. Wasserbaech ${ }^{66}$, J. Wood, F. Würthwein, A. Yagil, G. Zevi Della Porta

University of California, Santa Barbara - Department of Physics, Santa Barbara, U.S.A.

N. Amin, R. Bhandari, J. Bradmiller-Feld, C. Campagnari, M. Citron, A. Dishaw, V. Dutta, M. Franco Sevilla, L. Gouskos, R. Heller, J. Incandela, A. Ovcharova, H. Qu, J. Richman, D. Stuart, I. Suarez, S. Wang, J. Yoo

California Institute of Technology, Pasadena, U.S.A.

D. Anderson, A. Bornheim, J.M. Lawhorn, H.B. Newman, T.Q. Nguyen, M. Spiropulu, J.R. Vlimant, R. Wilkinson, S. Xie, Z. Zhang, R.Y. Zhu

Carnegie Mellon University, Pittsburgh, U.S.A.

M.B. Andrews, T. Ferguson, T. Mudholkar, M. Paulini, M. Sun, I. Vorobiev, M. Weinberg University of Colorado Boulder, Boulder, U.S.A.

J.P. Cumalat, W.T. Ford, F. Jensen, A. Johnson, M. Krohn, E. MacDonald, T. Mulholland, R. Patel, K. Stenson, K.A. Ulmer, S.R. Wagner

Cornell University, Ithaca, U.S.A.

J. Alexander, J. Chaves, Y. Cheng, J. Chu, A. Datta, K. Mcdermott, N. Mirman, J.R. Patterson, D. Quach, A. Rinkevicius, A. Ryd, L. Skinnari, L. Soffi, S.M. Tan, Z. Tao, J. Thom, J. Tucker, P. Wittich, M. Zientek

Fermi National Accelerator Laboratory, Batavia, U.S.A.

S. Abdullin, M. Albrow, M. Alyari, G. Apollinari, A. Apresyan, A. Apyan, S. Banerjee, L.A.T. Bauerdick, A. Beretvas, J. Berryhill, P.C. Bhat, G. Bolla ${ }^{\dagger}$, K. Burkett, J.N. Butler, A. Canepa, G.B. Cerati, H.W.K. Cheung, F. Chlebana, M. Cremonesi, J. Duarte, V.D. Elvira, J. Freeman, Z. Gecse, E. Gottschalk, L. Gray, D. Green, S. Grünendahl, 
O. Gutsche, J. Hanlon, R.M. Harris, S. Hasegawa, J. Hirschauer, Z. Hu, B. Jayatilaka, S. Jindariani, M. Johnson, U. Joshi, B. Klima, M.J. Kortelainen, B. Kreis, S. Lammel, D. Lincoln, R. Lipton, M. Liu, T. Liu, J. Lykken, K. Maeshima, J.M. Marraffino, D. Mason, P. McBride, P. Merkel, S. Mrenna, S. Nahn, V. O’Dell, K. Pedro, C. Pena, O. Prokofyev, G. Rakness, L. Ristori, A. Savoy-Navarro ${ }^{67}$, B. Schneider, E. Sexton-Kennedy, A. Soha, W.J. Spalding, L. Spiegel, S. Stoynev, J. Strait, N. Strobbe, L. Taylor, S. Tkaczyk, N.V. Tran, L. Uplegger, E.W. Vaandering, C. Vernieri, M. Verzocchi, R. Vidal, M. Wang, H.A. Weber, A. Whitbeck

University of Florida, Gainesville, U.S.A.

D. Acosta, P. Avery, P. Bortignon, D. Bourilkov, A. Brinkerhoff, L. Cadamuro, A. Carnes, M. Carver, D. Curry, R.D. Field, S.V. Gleyzer, B.M. Joshi, J. Konigsberg, A. Korytov, K.H. Lo, P. Ma, K. Matchev, H. Mei, G. Mitselmakher, D. Rosenzweig, K. Shi, D. Sperka, J. Wang, S. Wang, X. Zuo

Florida International University, Miami, U.S.A.

Y.R. Joshi, S. Linn

Florida State University, Tallahassee, U.S.A.

A. Ackert, T. Adams, A. Askew, S. Hagopian, V. Hagopian, K.F. Johnson, T. Kolberg, G. Martinez, T. Perry, H. Prosper, A. Saha, C. Schiber, R. Yohay

Florida Institute of Technology, Melbourne, U.S.A.

M.M. Baarmand, V. Bhopatkar, S. Colafranceschi, M. Hohlmann, D. Noonan, M. Rahmani, T. Roy, F. Yumiceva

\section{University of Illinois at Chicago (UIC), Chicago, U.S.A.}

M.R. Adams, L. Apanasevich, D. Berry, R.R. Betts, R. Cavanaugh, X. Chen, S. Dittmer, O. Evdokimov, C.E. Gerber, D.A. Hangal, D.J. Hofman, K. Jung, J. Kamin, C. Mills, I.D. Sandoval Gonzalez, M.B. Tonjes, H. Trauger, N. Varelas, H. Wang, X. Wang, Z. Wu, J. Zhang

The University of Iowa, Iowa City, U.S.A.

M. Alhusseini, B. Bilki ${ }^{68}$, W. Clarida, K. Dilsiz ${ }^{69}$, S. Durgut, R.P. Gandrajula, M. Haytmyradov, V. Khristenko, J.-P. Merlo, A. Mestvirishvili, A. Moeller, J. Nachtman, H. Ogul ${ }^{70}$, Y. Onel, F. Ozok ${ }^{71}$, A. Penzo, C. Snyder, E. Tiras, J. Wetzel

Johns Hopkins University, Baltimore, U.S.A.

B. Blumenfeld, A. Cocoros, N. Eminizer, D. Fehling, L. Feng, A.V. Gritsan, W.T. Hung, P. Maksimovic, J. Roskes, U. Sarica, M. Swartz, M. Xiao, C. You

The University of Kansas, Lawrence, U.S.A.

A. Al-bataineh, P. Baringer, A. Bean, S. Boren, J. Bowen, A. Bylinkin, J. Castle, S. Khalil,

A. Kropivnitskaya, D. Majumder, W. Mcbrayer, M. Murray, C. Rogan, S. Sanders, E. Schmitz, J.D. Tapia Takaki, Q. Wang 
Kansas State University, Manhattan, U.S.A.

S. Duric, A. Ivanov, K. Kaadze, D. Kim, Y. Maravin, D.R. Mendis, T. Mitchell, A. Modak, A. Mohammadi, L.K. Saini, N. Skhirtladze

Lawrence Livermore National Laboratory, Livermore, U.S.A.

F. Rebassoo, D. Wright

University of Maryland, College Park, U.S.A.

A. Baden, O. Baron, A. Belloni, S.C. Eno, Y. Feng, C. Ferraioli, N.J. Hadley, S. Jabeen, G.Y. Jeng, R.G. Kellogg, J. Kunkle, A.C. Mignerey, S. Nabili, F. Ricci-Tam, Y.H. Shin, A. Skuja, S.C. Tonwar, K. Wong

\section{Massachusetts Institute of Technology, Cambridge, U.S.A.}

D. Abercrombie, B. Allen, V. Azzolini, A. Baty, G. Bauer, R. Bi, S. Brandt, W. Busza, I.A. Cali, M. D'Alfonso, Z. Demiragli, G. Gomez Ceballos, M. Goncharov, P. Harris, D. Hsu, M. Hu, Y. Iiyama, G.M. Innocenti, M. Klute, D. Kovalskyi, Y.-J. Lee, P.D. Luckey, B. Maier, A.C. Marini, C. Mcginn, C. Mironov, S. Narayanan, X. Niu, C. Paus, C. Roland, G. Roland, G.S.F. Stephans, K. Sumorok, K. Tatar, D. Velicanu, J. Wang, T.W. Wang, B. Wyslouch, S. Zhaozhong

University of Minnesota, Minneapolis, U.S.A.

A.C. Benvenuti, R.M. Chatterjee, A. Evans, P. Hansen, S. Kalafut, Y. Kubota, Z. Lesko, J. Mans, N. Ruckstuhl, R. Rusack, J. Turkewitz, M.A. Wadud

University of Mississippi, Oxford, U.S.A.

J.G. Acosta, S. Oliveros

University of Nebraska-Lincoln, Lincoln, U.S.A.

E. Avdeeva, K. Bloom, D.R. Claes, C. Fangmeier, F. Golf, R. Gonzalez Suarez, R. Kamalieddin, I. Kravchenko, J. Monroy, J.E. Siado, G.R. Snow, B. Stieger

State University of New York at Buffalo, Buffalo, U.S.A.

A. Godshalk, C. Harrington, I. Iashvili, A. Kharchilava, C. Mclean, D. Nguyen, A. Parker, S. Rappoccio, B. Roozbahani

Northeastern University, Boston, U.S.A.

G. Alverson, E. Barberis, C. Freer, A. Hortiangtham, D.M. Morse, T. Orimoto, R. Teixeira De Lima, T. Wamorkar, B. Wang, A. Wisecarver, D. Wood

Northwestern University, Evanston, U.S.A.

S. Bhattacharya, O. Charaf, K.A. Hahn, N. Mucia, N. Odell, M.H. Schmitt, K. Sung, M. Trovato, M. Velasco

University of Notre Dame, Notre Dame, U.S.A.

R. Bucci, N. Dev, M. Hildreth, K. Hurtado Anampa, C. Jessop, D.J. Karmgard, N. Kellams, K. Lannon, W. Li, N. Loukas, N. Marinelli, F. Meng, C. Mueller, Y. Musienko ${ }^{34}$, M. Planer, A. Reinsvold, R. Ruchti, P. Siddireddy, G. Smith, S. Taroni, M. Wayne, A. Wightman, M. Wolf, A. Woodard 
The Ohio State University, Columbus, U.S.A.

J. Alimena, L. Antonelli, B. Bylsma, L.S. Durkin, S. Flowers, B. Francis, A. Hart, C. Hill, W. Ji, T.Y. Ling, W. Luo, B.L. Winer

Princeton University, Princeton, U.S.A.

S. Cooperstein, P. Elmer, J. Hardenbrook, S. Higginbotham, A. Kalogeropoulos, D. Lange, M.T. Lucchini, J. Luo, D. Marlow, K. Mei, I. Ojalvo, J. Olsen, C. Palmer, P. Piroué, J. Salfeld-Nebgen, D. Stickland, C. Tully

University of Puerto Rico, Mayaguez, U.S.A.

S. Malik, S. Norberg

Purdue University, West Lafayette, U.S.A.

A. Barker, V.E. Barnes, S. Das, L. Gutay, M. Jones, A.W. Jung, A. Khatiwada, B. Mahakud, D.H. Miller, N. Neumeister, C.C. Peng, S. Piperov, H. Qiu, J.F. Schulte, J. Sun, F. Wang, R. Xiao, W. Xie

Purdue University Northwest, Hammond, U.S.A.

T. Cheng, J. Dolen, N. Parashar

Rice University, Houston, U.S.A.

Z. Chen, K.M. Ecklund, S. Freed, F.J.M. Geurts, M. Kilpatrick, W. Li, B.P. Padley, J. Roberts, J. Rorie, W. Shi, Z. Tu, J. Zabel, A. Zhang

University of Rochester, Rochester, U.S.A.

A. Bodek, P. de Barbaro, R. Demina, Y.t. Duh, J.L. Dulemba, C. Fallon, T. Ferbel, M. Galanti, A. Garcia-Bellido, J. Han, O. Hindrichs, A. Khukhunaishvili, P. Tan, R. Taus

Rutgers, The State University of New Jersey, Piscataway, U.S.A.

A. Agapitos, J.P. Chou, Y. Gershtein, E. Halkiadakis, M. Heindl, E. Hughes, S. Kaplan, R. Kunnawalkam Elayavalli, S. Kyriacou, A. Lath, R. Montalvo, K. Nash, M. Osherson, H. Saka, S. Salur, S. Schnetzer, D. Sheffield, S. Somalwar, R. Stone, S. Thomas, P. Thomassen, M. Walker

University of Tennessee, Knoxville, U.S.A.

A.G. Delannoy, J. Heideman, G. Riley, S. Spanier

Texas A\&M University, College Station, U.S.A.

O. Bouhali ${ }^{72}$, A. Celik, M. Dalchenko, M. De Mattia, A. Delgado, S. Dildick, R. Eusebi,

J. Gilmore, T. Huang, T. Kamon ${ }^{73}$, S. Luo, R. Mueller, A. Perloff, L. Perniè, D. Rathjens,

A. Safonov

Texas Tech University, Lubbock, U.S.A.

N. Akchurin, J. Damgov, F. De Guio, P.R. Dudero, S. Kunori, K. Lamichhane, S.W. Lee, T. Mengke, S. Muthumuni, T. Peltola, S. Undleeb, I. Volobouev, Z. Wang

Vanderbilt University, Nashville, U.S.A.

S. Greene, A. Gurrola, R. Janjam, W. Johns, C. Maguire, A. Melo, H. Ni, K. Padeken, J.D. Ruiz Alvarez, P. Sheldon, S. Tuo, J. Velkovska, M. Verweij, Q. Xu 


\section{University of Virginia, Charlottesville, U.S.A.}

M.W. Arenton, P. Barria, B. Cox, R. Hirosky, M. Joyce, A. Ledovskoy, H. Li, C. Neu, T. Sinthuprasith, Y. Wang, E. Wolfe, F. Xia

\section{Wayne State University, Detroit, U.S.A.}

R. Harr, P.E. Karchin, N. Poudyal, J. Sturdy, P. Thapa, S. Zaleski

\section{University of Wisconsin - Madison, Madison, WI, U.S.A.}

M. Brodski, J. Buchanan, C. Caillol, D. Carlsmith, S. Dasu, L. Dodd, B. Gomber, M. Grothe, M. Herndon, A. Hervé, U. Hussain, P. Klabbers, A. Lanaro, K. Long, R. Loveless, T. Ruggles, A. Savin, V. Sharma, N. Smith, W.H. Smith, N. Woods

\section{t: Deceased}

1: Also at Vienna University of Technology, Vienna, Austria

2: Also at IRFU, CEA, Université Paris-Saclay, Gif-sur-Yvette, France

3: Also at Universidade Estadual de Campinas, Campinas, Brazil

4: Also at Federal University of Rio Grande do Sul, Porto Alegre, Brazil

5: Also at Université Libre de Bruxelles, Bruxelles, Belgium

6: Also at University of Chinese Academy of Sciences, Beijing, China

7: Also at Institute for Theoretical and Experimental Physics, Moscow, Russia

8: Also at Joint Institute for Nuclear Research, Dubna, Russia

9: Also at Cairo University, Cairo, Egypt

10: Now at Helwan University, Cairo, Egypt

11: Also at Zewail City of Science and Technology, Zewail, Egypt

12: Also at Department of Physics, King Abdulaziz University, Jeddah, Saudi Arabia

13: Also at Université de Haute Alsace, Mulhouse, France

14: Also at Skobeltsyn Institute of Nuclear Physics, Lomonosov Moscow State University, Moscow, Russia

15: Also at CERN, European Organization for Nuclear Research, Geneva, Switzerland

16: Also at RWTH Aachen University, III. Physikalisches Institut A, Aachen, Germany

17: Also at University of Hamburg, Hamburg, Germany

18: Also at Brandenburg University of Technology, Cottbus, Germany

19: Also at MTA-ELTE Lendület CMS Particle and Nuclear Physics Group, Eötvös Loránd University, Budapest, Hungary

20: Also at Institute of Nuclear Research ATOMKI, Debrecen, Hungary

21: Also at Institute of Physics, University of Debrecen, Debrecen, Hungary

22: Also at Indian Institute of Technology Bhubaneswar, Bhubaneswar, India

23: Also at Institute of Physics, Bhubaneswar, India

24: Also at Shoolini University, Solan, India

25: Also at University of Visva-Bharati, Santiniketan, India

26: Also at Isfahan University of Technology, Isfahan, Iran

27: Also at Plasma Physics Research Center, Science and Research Branch, Islamic Azad University, Tehran, Iran

28: Also at Università degli Studi di Siena, Siena, Italy

29: Also at Kyunghee University, Seoul, Korea

30: Also at International Islamic University of Malaysia, Kuala Lumpur, Malaysia

31: Also at Malaysian Nuclear Agency, MOSTI, Kajang, Malaysia 
32: Also at Consejo Nacional de Ciencia y Tecnología, Mexico city, Mexico

33: Also at Warsaw University of Technology, Institute of Electronic Systems, Warsaw, Poland

34: Also at Institute for Nuclear Research, Moscow, Russia

35: Now at National Research Nuclear University 'Moscow Engineering Physics Institute' (MEPhI), Moscow, Russia

36: Also at St. Petersburg State Polytechnical University, St. Petersburg, Russia

37: Also at University of Florida, Gainesville, U.S.A.

38: Also at P.N. Lebedev Physical Institute, Moscow, Russia

39: Also at California Institute of Technology, Pasadena, U.S.A.

40: Also at Budker Institute of Nuclear Physics, Novosibirsk, Russia

41: Also at Faculty of Physics, University of Belgrade, Belgrade, Serbia

42: Also at INFN Sezione di Pavia ${ }^{a}$, Università di Pavia ${ }^{b}$, Pavia, Italy

43: Also at University of Belgrade, Faculty of Physics and Vinca Institute of Nuclear Sciences, Belgrade, Serbia

44: Also at Scuola Normale e Sezione dell'INFN, Pisa, Italy

45: Also at National and Kapodistrian University of Athens, Athens, Greece

46: Also at Riga Technical University, Riga, Latvia

47: Also at Universität Zürich, Zurich, Switzerland

48: Also at Stefan Meyer Institute for Subatomic Physics (SMI), Vienna, Austria

49: Also at Adiyaman University, Adiyaman, Turkey

50: Also at Istanbul Aydin University, Istanbul, Turkey

51: Also at Mersin University, Mersin, Turkey

52: Also at Piri Reis University, Istanbul, Turkey

53: Also at Gaziosmanpasa University, Tokat, Turkey

54: Also at Ozyegin University, Istanbul, Turkey

55: Also at Izmir Institute of Technology, Izmir, Turkey

56: Also at Marmara University, Istanbul, Turkey

57: Also at Kafkas University, Kars, Turkey

58: Also at Istanbul University, Faculty of Science, Istanbul, Turkey

59: Also at Istanbul Bilgi University, Istanbul, Turkey

60: Also at Hacettepe University, Ankara, Turkey

61: Also at Rutherford Appleton Laboratory, Didcot, United Kingdom

62: Also at School of Physics and Astronomy, University of Southampton, Southampton, United Kingdom

63: Also at Monash University, Faculty of Science, Clayton, Australia

64: Also at Bethel University, St. Paul, U.S.A.

65: Also at Karamanoğlu Mehmetbey University, Karaman, Turkey

66: Also at Utah Valley University, Orem, U.S.A.

67: Also at Purdue University, West Lafayette, U.S.A.

68: Also at Beykent University, Istanbul, Turkey

69: Also at Bingol University, Bingol, Turkey

70: Also at Sinop University, Sinop, Turkey

71: Also at Mimar Sinan University, Istanbul, Istanbul, Turkey

72: Also at Texas A\&M University at Qatar, Doha, Qatar

73: Also at Kyungpook National University, Daegu, Korea 